\title{
MHD effect on unsteady flow of tangent hyperbolic nano-fluid past a moving cylinder with chemical reaction
}

\author{
Partha Protim Gharami ${ }^{1}$-Sk. Reza-E-Rabbi ${ }^{2}$ D . S. M. Arifuzzaman ${ }^{3}$ (D) Md. Shakhaoath Khan $^{4}$ (D) Tanmoy Sarkar $^{1}$. \\ Sarder Firoz Ahmmed ${ }^{1}$
}

Received: 5 January 2020 / Accepted: 10 June 2020 / Published online: 20 June 2020

(c) Springer Nature Switzerland AG 2020

\begin{abstract}
This study presents the exploration of unsteady magnetohydrodynamic (MHD) free convection flow of tangent hyperbolic nano-fluid flow on a moving cylinder with Brownian motion and thermophoresis effects. The current flow analysis yields nonlinear partial differential equations. The governing equations such as continuity, momentum, temperature and concentration are converted into dimensionless form and then solved numerically by adopting explicit finite difference method where Compaq Visual FORTRAN 6.6.a was also used for simulating the fluid flow system. The numerical outcomes are showed graphically to understand the result clearly. For the accurateness of the numerical technique a stability and convergence analysis was carried out. The aim was to illustrate the physical impacts of chemical reaction parameter, thermal radiation and viscous dissipation on various fluid fields along with the advanced visualization through streamlines. By comparing with the previous studies it was found that this fluid influenced the mass and heat properties more significantly rather than the other fluid. Additionally, this model predicts the shear thinning attitude significantly and describes the blood flow accurately. It has also applications in biological sciences, bio-engineering maneuver, and petroleum industries. Eventually the obtained outcomes were validated with previously published articles.
\end{abstract}

Keywords Tangent hyperbolic fluid · Nanoparticle $\cdot$ EFDM $\cdot$ Chemical reaction · MHD

\begin{tabular}{|c|c|c|c|}
\hline & $K r$ & Chemical reaction parameter (-) \\
\hline \multicolumn{2}{|c|}{$B_{0} \quad$ External magnetic field $\left(\mathrm{Wb} \mathrm{m}^{-2}\right)$} & Le & Lewis number $(-)$ \\
\hline$C_{p}$ & Specific heat at constant pressure $\left(\mathrm{J} \mathrm{kg}^{-1} \mathrm{~K}^{-1}\right)$ & $M$ & Magnetic parameter (-) \\
\hline C & Concentration component & $n$ & Power low index (-) \\
\hline$C_{w}$ & Concentration of the cylinder (mol.) & $N b$ & Brownian parameter (-) \\
\hline$C_{\infty}$ & Concentration away from the cylinder & $N t$ & Thermophoresis parameter (-) \\
\hline$C_{f}$ & Skin friction $(-)$ & $\mathrm{Nu}$ & Nusselt number (-) \\
\hline$D_{B}$ & Brownian diffusion coefficient & $\operatorname{Pr}$ & Prandlt number $(-)$ \\
\hline$D_{T}$ & Thermophoresis diffusion coefficient & $q_{r}$ & Radiative heat flux $\left(\mathrm{kg} \mathrm{m}^{-2}\right)$ \\
\hline$g$ & Gravitational acceleration $\left(\mathrm{m} \mathrm{s}^{-2}\right)$ & Ra & Radiation parameter (-) \\
\hline Gr & Thermal Grashof number (-) & Sh & Sherwood number (-) \\
\hline Gm & Mass Grashof number (-) & $T$ & Temperature (K) \\
\hline
\end{tabular}

$\triangle$ Sk. Reza-E-Rabbi, rabbi06@uap-bd.edu; Partha Protim Gharami, gharamipartha48@gmail.com; S. M. Arifuzzaman, 19243001@student.westernsydney.edu.au; Md. Shakhaoath Khan, ms.khan@monash.edu; Tanmoy Sarkar, tanmoy.ku11@gmail.com; Sarder Firoz Ahmmed, sfahmmed@yahoo.com | ${ }^{1}$ Mathematics Discipline, Khulna University, Khulna 9208, Bangladesh.

${ }^{2}$ Department of Basic Sciences \& Humanities, University of Asia Pacific, Dhaka 1205, Bangladesh. ${ }^{3}$ Centre for Infrastructure Engineering, Western Sydney University, Sydney, NSW 2751, Australia. ${ }^{4}$ Department of Chemical Engineering, Monash University, Clayton, VIC 3800, Australia. 
$T_{\infty} \quad$ Temperature away from the cylinder

$T_{w} \quad$ Temperature of the fluid

$\bar{t} \quad$ Dimensional time (s)

$t \quad$ Non-dimensional time (-)

$u_{0} \quad$ Uniform velocity $\left(\mathrm{m} \mathrm{s}^{-1}\right)$

$u, v \quad$ Dimensional velocity of the fluid in $x$ and $r$ direction

$U \quad$ Non-dimensional velocity (-)

We Weissenberg number

$X, R \quad$ Non-dimensional coordinate axis along and normal to the cylinder

$x, r \quad$ Coordinate axis along and normal to the cylinder

$\theta \quad$ Non-dimensional temperature (-)

$\phi \quad$ Dimensionless concentration (-)

\section{Greek symbols}

$v \quad$ Kinematic viscosity $\left(\mathrm{m}^{2} \mathrm{~s}^{-1}\right)$

$\kappa \quad$ Thermal conductivity $\left(\mathrm{W} \mathrm{m}^{-1} \mathrm{~K}^{-1}\right)$

$\beta_{T} \quad$ Thermal expansion coefficient (1/K)

$\beta_{C} \quad$ Concentration expansion coefficient

$\sigma_{s} \quad$ Stefan-Boltzmann constant $\left(\mathrm{W} \mathrm{m} \mathrm{K}^{-4}\right)$

$\rho \quad$ Density $\left(\mathrm{kg} \mathrm{m}^{-3}\right)$

$\Gamma \quad$ Rate time constant (s)

\section{Abbreviations}

EFDM Explicit finite difference method

MHD Magnetohydrodynamic

\section{Introduction}

Over the past three decades due to multi-purpose uses in modern high-tech industries, nanofluid flow research has received considerable attention. Many researchers have studied nanofluids both experimentally and theoretically. They conclude that nanofluids upsurge the updraft conductivity of convection fluid. They have restricted updraft conductivity properties. Because of its enormous solicitations in different production processes, for example polymers, textiles, nourishment industries, etc., the boundary layer stream of non-Newtonian liquids affected by the unstable cylinder has been tested for many years. Capable of cutting thinning phenomena, tangent fluid is pseudoplastic exemplary of four constants. When the pour drops with an accumulative rate of shear stress, the hyperbolic fluid trials conflict of liquid. The most common hyperbolic liquors are nail polish, tomato sauce, blood, varnish and whipped cream etc.

The influence of heat generation/absorption on tangent hyperbolic nano-fluid near the stagnation point over a stretching cylinder has been studied by Salahuddin et al. [1]. The governing partial differential equations are transformed into ordinary differential equation and the ordinary differential equation has been solved numerically by using shooting method. Ibrahim [2] presented the effects of thermal radiation on magneto-hydrodynamic flow of tangent hyperbolic fluid with nanoparticle past an enlarging sheet with second order slip and convective boundary condition. Similarity transformation has been considered to solve the governing non-linear boundary-value problem into coupled higher order non-linear ordinary differential equations. Then the equations are solved via matlab bvp4c function. Khan et al. [3], they analyzed two dimensional flow of MHD hyperbolic tangent fluid with nanoparticles towards a stretching surface. The nonlinear set of partial differential equations has been reduced into ordinary differential equations by using similarity transformation and solved numerically by using shooting method and additionally local skin friction, local Nusselt number and local Sherwood number has been computed and analyzed in their research work. Hayat et al. [4], calculated combined effects of Soret (thermal diffusion) and Dufour (diffusionthermo) in MHD stagnation point flow of tangent hyperbolic fluid by a stretching sheet. Similarity transformation is used to convert the nonlinear partial differential equations into ordinary differential equations. The series solutions of boundary layer equations along with boundary conditions are studied in their research work. Hayat et al. [5], premeditated the inspiration of influence of applied magnetic field in peristalsis of hyperbolic tangent hyperbolic nanofluid. The relevant equations are simplified by applying long wave length assumption. The graphical and physical clarification for velocity, temperature, concentration and heat transfer rate are examined through ND Solve command and MATHEMATICA. Khan et al. [6] studied the effects of chemical reactive species and solar radiation on Carreau nanofluid induced by a stretching sheet with variable thickness. Runge-Kutta Fehlberg method for numerical solutions of the dimensionless governing equations has been used in their experiment. Hayat et al. [7] explored magnetic nanoparticles in stretched flow of tangent hyperbolic nanofluid. In their research work, nonlinear nanofluid model due to Brownian motion and thermophoresis mechanisms has been used and a mathematical formulation is modeled employing boundary layer concept. Kumar et al. [8] studied an unsteady squeezed flow of a tangent hyperbolic fluid over a sensor surface in the presence of variable thermal conductivity. The partial non-linear differential equation has been converted into ordinary differential equations by using similarity transformation. The transformed differential equations are solved numerically by using RKF45 technique. Malik et al. [9] obtained a numerical solution of MHD flow of tangent hyperbolic fluid model over a stretching cylinder. Magneto hydrodynamics is a phenomenon in which the conducting fluid particles interact with electromagnetic fields. In their research work the governing boundary layer equation of tangent hyperbolic fluid 
is transformed into ordinary differential equation using similarity transformation and then it was solved numerically by applying the implicit finite difference method with Keller box scheme. The impact of thermal radiation on boundary layer flow of dusty hyperbolic tangent fluid over a stretching sheet in the presence of magnetic field is examined by Kumar et al. [10]. Similarity transformation is used to convert the partial differential equations into a nonlinear ordinary differential equation and solved by applying Runge-Kutta-Fehlberg forth-fifth order (RKF45 method) with the help of shooting technique. Hussain et al. [11], analyzed thermos-physical aspects of MHD tangent hyperbolic fluid flow over a non-linear stretching sheet with viscous dissipation and convective boundary conditions. Similarity variables are used to convert PDE into ODE and solved with homotopic analysis. Pseudo plasticity is the basic reason of divergence from Newtonian fluid to non-Newtonian fluid, because non-Newtonian fluids have variable viscosity due to applied force.

Nanofluid is a specific class of fluids suspended by the 1-1000 nm size particles which have highly enhanced thermal properties. Heat and mass transfer of Williamson nano-fluid flow yield by an inclined Lorentz force over a nonlinear stretching sheet was discussed by Khan et al. [12]. Similarity transformation is used to reduce the partial form of the equation into ordinary form and solved numerically by using shooting method. Rahman et al. [13] introduced hydro-magnetic slip flow of water based nanofluids past a wedge with convective surface in the presence of heat generation (or) absorption. Behavior of Thermal radiation effects on Williamson fluid flow due to an expanding/ contracting cylinder with nano-materials is explored by Hashim et al. [14]. Transpiration effect on stagnation-point flow of a Carreau nanofluid in the presence of thermophoresis and Brownian motion is examined by Sulochana et al. [15]. Mamatha et al. [16], analyzed heat and mass flux conditions on magneto hydrodynamic unsteady Eyring-Powel dusty nanofluid over a sheet. Runge-Kuttai system is used to get mathematical explanation in their investigation. Radiation and chemical reaction effects on MHD Casson fluid flow past an oscillating vertical plate embedded in porous medium is introduced by Hari and Harshad [17]. Laplace transform is performed to solve administering equation. Both isothermal and ramped wall temperature were taken into account in their experiment. Rabbi et al. [18] studied hydro magnetic Casson nanofluid flow through a stretching plane surface. Their analysis depicted the heat and mass transport phenomena for both stretching and shrinking cases. Explicit finite scheme together with stability and convergence analysis were also discussed in their investigation. However, in recent time lots of researchers have carried out their work by considering the above discussed explicit technique. Various types of Newtonian and
non-Newtonian fluids had been investigated by considering different physical aspects. For details, one can refer the following articles $[19,20]$. Combined techniques of nanoparticles and wavy duct have been studied by Ma et al. [21]. It is been seen that on a wavy surface entropy generation acts as a reducing function of nanoparticle concentration. Hsiao [22] studied energy conversion problem for electric hydro magnetic heat and mass mixed convection in an incompressible second grade Maxwell fluid with radiation and viscous dissipation effects. Similarity transformation and finite difference method have been imposed to solve the governing equations numerically. A coupled radiation heating was introduced in this work. Second law management for iron oxide nanofluid inside a porous plate is contemplated by Sheikholeslami [23]. Due to the reduction of magnetic force and permeability exergy drop and Bejan number got detract. Heat transmission augmentation through nanofluid is investigated by Qi et al. [24]. The influence of rotated twisting tape has been considered. In a circular tube the exergy efficiency was found in a significant way with this twisted tape. However, Zhao et al. [25] did an experimental work with the exergy efficiency of cylindrical grooves. Additionally, entropy generation and thermal efficiency were also considered.

Getting motivated by the work of Salahuddin et al. [1] and Hayat et al. [7], the present analysis explored the impression of unsteady magneto hydrodynamic (MHD) free convection flow of tangent hyperbolic nano-fluid impinging on a moving cylinder with Brownian motion and thermophoresis effects. The mentioned authors depicted their work on usual flow filed by considering the impact of three or four parameters. The physical impacts of chemical reaction parameter, thermal radiation and viscous dissipation were unexplored by them, which is explored in this paper. However, in addition the advanced visualisation of the fluid flow has also been depicted newly in this paper through streamlines. The novel aspect of this work is to make a comparative study between present and previous study and observe that the fluid influence in the thermal and mass properties are more significant in the present study. However, one of the major cause is the mentioned fluid has extensive application in metallurgy, penetrating processes, biological sciences, bioengineering maneuver, geophysics, chemical and petroleum industries. Additionally, the tangent hyperbolic fluid model is one of the important fluid model in the group of non-Newtonian fluid models. From laboratory experiments it is found that this model predicts shear thinning phenomenon very precisely. Additionally, this model describes the blood flow very accurately. In this work, firstly, a mathematical model has been established by considering some physical aspects. Then in the next section the dimensionless form of the fundamental equations are solved by imposing explicit scheme followed by the stability and convergence analysis. Further, 
a result and discussion section has been presented precisely with graphical illustration and eventually a concluding section has been depicted briefly. For grid independency test an appendix section has also been given lastly.

\section{Mathematical model}

In this investigation, a tangent hyperbolic unstable twodimensional progression of incompressible nanofluid flow over an extensible cylinder has been illustrated. Here, stream is thought to be located at area $r>0$. Cylinder is placed at $r=R$, here; $r$ is harmonizing normal to cylinder. Flow is produced by activity of two fold equivalent and contrasting strength laterally $x$ axis with velocity $u$ and $v$ is normal to the flow in $r$ direction. An identical magnetized field $B_{o}$ is executed along $r$ axis and cylinder being enlarged along $x$ axis with velocity. $T_{\infty}$ and $C_{\infty}$ are considered surrounding temperature and surrounding concentration correspondingly. It is seen from the experiment that no particle motion is present at surface and influence of Brownian motion and thermophoresis are considered in the energy and concentration principal equations. The wall temperature and wall concentration are controlled by $T_{w}$ and $C_{w}$ respectively (See Fig. 1).

The heat Radiative flux is assumed as:

$q_{r}=\frac{4 \sigma_{*}}{3 k^{*}} \frac{\partial T^{4}}{\partial r}$

Here, $k^{*}$ and $\sigma_{*}$ are Rossseland's factor and StefanBoltzmann constant respectively where expansion of $T^{4}$ about $T_{0}$ can be described by utilizing Taylor sequence as,

$T^{4} \cong 4 T_{0}^{3} T-3 T_{0}^{4}$

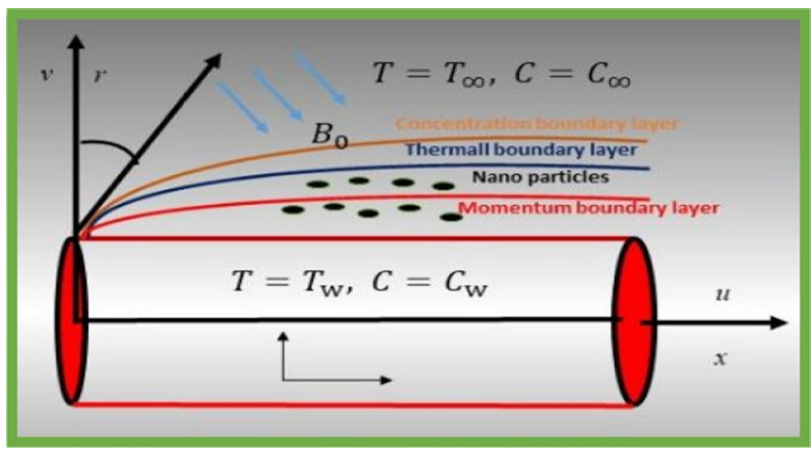

Fig. 1 Physical model and co-ordinate system (Salahuddin et al. [1]; Malik et al. [9])
Constitutive equation of hyperbolic fluid is seen through Eq. (3)

$\tau=\left[\mu_{0}+\left(\mu_{0}+\mu_{\infty}\right) \tanh (\Gamma \bar{\gamma})^{n}\right] \bar{\gamma} \quad($ Akbar et al. 2013)

In the above equation $n$ represents power law index, $\tau$ represents stress tensor, $\mu_{\infty}$ represents viscosity of immeasurable shear rate, $\mu_{0}$ is rate with zero viscosity, $\Gamma$ Williamson factor and symbol $\bar{\gamma}$ can be defined in the below form

$\overline{\dot{\gamma}}=\frac{1}{2} \sqrt{\frac{1}{2} \sum_{i} \sum_{j} \dot{\bar{\gamma}}_{i j}} \overline{\dot{\gamma}}_{j i}=\sqrt{\frac{1}{2} \Pi}$

It isn't thinkable to deliberate the matter when immeasurable shear level viscidness $\mu_{\infty}=0$ for the Eq. (4) and $\Gamma \bar{\gamma}<1$. Then Eq. (4) can be written as

$\tau=\mu_{0}\left[(\Gamma \bar{\gamma})^{n}\right]=\mu_{0}[1+n(\Gamma \bar{\gamma}-1) \bar{\gamma}$

Now it is time to discuss our main mathematical model i.e. governing equations which is formed by taking continuity, momentum, energy and concentration equations. These four equations with boundary layer approximation are given below (Salahuddin et al. [1]; Hayat et al. [7]):

$\frac{\partial(r u)}{\partial x}+\frac{\partial(r v)}{\partial r}=0$

$\frac{\partial u}{\partial \bar{t}}+u \frac{\partial u}{\partial x}+v \frac{\partial u}{\partial r}=v\left[(1-n) \frac{\partial^{2} u}{\partial r^{2}}+(1-n) \frac{1}{r} \frac{\partial u}{\partial r}\right.$

$$
\left.+n \sqrt{2} \Gamma \frac{\partial u}{\partial r} \frac{\partial^{2} u}{\partial r^{2}}+\frac{n \Gamma}{\sqrt{2} r}\left(\frac{\partial u}{\partial r}\right)^{2}\right]-\frac{\sigma B_{0}^{2}}{\rho} u
$$$$
+g \beta_{T}\left(T-T_{\infty}\right)+g \beta_{C}\left(C-C_{\infty}\right)
$$

$$
\begin{aligned}
\frac{\partial T}{\partial \bar{t}} & +u \frac{\partial T}{\partial x}+v \frac{\partial T}{\partial r}=\frac{K}{\rho C_{p} r} \frac{\partial}{\partial r}\left(r \frac{\partial T}{\partial r}\right) \\
& +\frac{\sigma B_{0}^{2} \bar{u}^{2}}{\rho C_{p}}+\frac{\tau}{\rho C_{p}}\left\{D_{B} \frac{\partial C}{\partial r} \frac{\partial T}{\partial r}+\frac{D_{T}}{T_{\infty}}\left[\left(\frac{\partial T}{\partial r}\right)^{2}\right]\right\} \\
& -\frac{1}{\rho C_{p} r} \frac{\partial}{\partial r}\left(r q_{r}\right)+\frac{\lambda}{\rho C_{p}}\left(T-T_{\infty}\right)
\end{aligned}
$$

$\frac{\partial C}{\partial \bar{t}}+u \frac{\partial C}{\partial x}+v \frac{\partial C}{\partial r}=\frac{D_{B}}{r} \frac{\partial}{\partial r}\left(r \frac{\partial C}{\partial r}\right)+\frac{D_{T}}{T_{\infty}} \frac{1}{r} \frac{\partial}{\partial r}\left(r \frac{\partial T}{\partial r}\right)-K r\left(C-C_{\infty}\right)$

where $u, v$ represents velocity in $x$ and $r$ direction individually, $T$ be fluid temperature, $C$ be fluid concentration and $G r, G m, \rho, C_{p}, \beta_{T}, \beta_{C} v$,n denotes thermal Grashof number, modified Grashof number, fluid density, specific heat capacity, volumetric expansion for temperature, volumetric expansion for concentration, kinematic viscosity and power law index respectively. $\lambda$ denotes heat source when $\lambda>0$ and heat sink when $\lambda<0, D_{T}$ be thermophoresis 
diffusion coefficient, $\Gamma$ be Williamson factor, $D_{B}$ be Brownian diffusion coefficient.

Then associated frontier conditions are

$\bar{t} \leq 0: u=0, v=0, T=T_{\infty}, C=C_{\infty}$ for all $x \geq 0$ and $r \geq 0$

$\bar{t} \geq 0: u=u_{0}, v=0, T=T_{w}, C=C_{w} \quad$ at $r=r_{0}$

$u=0, v=0, T=T_{\infty}, C=C_{\infty} \quad$ at $x=0$ and $r \geq r_{0}$

$u \rightarrow 0, T \rightarrow T_{\infty}, C \rightarrow C_{\infty}$ as $r \rightarrow \infty$
The physical non-dimensional quantities such that specific skin frictions, Nusselt and Sherwood numbers are depicted as,

$$
\begin{aligned}
& C_{f}=-\frac{1}{2 \sqrt{2}} G r^{-3 / 4}\left(\frac{\partial U}{\partial R}\right)_{R=0} \\
& N u=\frac{1}{\sqrt{2}} G r^{-3 / 4}\left(\frac{\partial T}{\partial R}\right)_{R=0}
\end{aligned}
$$

$X=\frac{x v}{u_{0} r_{0}^{2}}, R=\frac{r}{r_{0}}, U=\frac{u}{u_{0}}, V=\frac{v r_{0}}{v}, t=\frac{\bar{t} v}{r_{0}^{2}}, \theta=\frac{T-T_{\infty}}{T_{w}-T_{\infty}}, \phi=\frac{C-C_{\infty}}{C_{w}-C_{\infty}}$

By applying Eq. (10), the governing equation i.e. continuity, momentum, energy and concentration eqs. are attained as

$\frac{\partial(R U)}{\partial X}+\frac{\partial(R V)}{\partial R}=0$

$\frac{\partial U}{\partial t}+U \frac{\partial U}{\partial X}+V \frac{\partial U}{\partial R}=(1-n)\left[\frac{\partial^{2} U}{\partial R^{2}}+\frac{1}{R} \frac{\partial U}{\partial R}\right]+W e\left[\frac{\partial U}{\partial R} \frac{\partial^{2} U}{\partial R^{2}}+\frac{1}{2 R}\left(\frac{\partial U}{\partial R}\right)^{2}\right]+G r \theta+G m \phi-M U$

The stream function $\psi$ satisfies the continuity equation and connected with dimensionless velocityi components

$\frac{\partial \theta}{\partial t}+u \frac{\partial \theta}{\partial X}+V \frac{\partial \theta}{\partial R}=\frac{1}{\operatorname{Pr}}\left(1+\frac{4}{3 R a}\right)\left(\frac{\partial^{2} \theta}{\partial R^{2}}+\frac{1}{R} \frac{\partial \theta}{\partial R}\right)+N b \frac{\partial \theta}{\partial R} \frac{\partial \phi}{\partial R}-N t\left(\frac{\partial \theta}{\partial R}\right)^{2}+E c M U^{2}+Q \theta$

$\frac{\partial \theta}{\partial t}+U \frac{\partial \phi}{\partial X}+V \frac{\partial \phi}{\partial R}=\frac{1}{L e}\left[\left(\frac{\partial^{2} \phi}{\partial R^{2}}+\frac{1}{R} \frac{\partial \phi}{\partial R}\right)+\left(\frac{N t}{N b}\right)\left(\frac{\partial^{2} \theta}{\partial R^{2}}+\frac{1}{R} \frac{\partial \theta}{\partial R}\right)\right]-K r \phi$

The boundary condition becomes

$t \leq 0: U=0, V=0, \theta=0, \phi=0$ everywhere

$t>0: U=1, V=0, \theta=1, \phi=1 \quad$ at $R=1$

$U=0, V=0, \theta=0, \phi=0$ at $\mathrm{X}=0$ and $\mathrm{R} \geq 1$

$U \rightarrow 0, V \rightarrow 0, \theta \rightarrow 0, \phi \rightarrow 0$ as $R \rightarrow \infty$

where the acquired physical factors are, Weissenberg number, We $=\frac{\sqrt{2} n \Gamma u_{0}}{r_{0}}$, Magnetic factor, $M=\frac{\sigma B_{0}^{2} r_{0}^{2}}{\rho v}$, thermal Grashof number, $G r=\frac{g \beta_{T} r_{0}^{2}\left(T_{w}-T_{\infty}\right)}{u_{0} v}$, modified Grashof number, $G m=\frac{g \beta_{C} r_{0}^{2}\left(C_{w}-C_{\infty}\right)}{u_{0} v}$, Prandtl number, $\operatorname{Pr}=\frac{\rho c_{p} v}{K}$, radiationi factor, $R a=\frac{u_{e} v}{4 \sigma_{s} T_{\infty}^{3}}$, Heat generation/absorption factor, $Q=\frac{\lambda}{\rho c_{p}}$, Brownian factor, $N b=\frac{\tau D_{B}\left(C_{w}-C_{\infty}\right)}{\rho c_{p} v}$, thermophoresis ifactor, $N t=\frac{\tau D_{T}\left(T_{w}-T_{\infty}\right)}{\rho c_{p} v T_{\infty}}, \quad$ Lewis number, Le $=\frac{v}{D_{B}}$, $K e=$ mean absorption coefficient, chemical reaction factor, $K r=\frac{K c r_{0}^{2}}{v}$. in standard form as $U=\frac{\partial \psi}{\partial Y}, V=-\frac{\partial \psi}{\partial X}$.

\section{Numerical technique}

Here, the non-dimensional coupled differential Eqs. (11) to (15) are elucidated numerically by utilizing explicit finite difference technique. For finite difference analysis it is divided the tangent hyperbolic fluid flow into grid spaces which is analogous to $X$ and $R$ is perpendicular to cylinder. In this investigation, it is taken that the altitude of the cylinder $X_{\max }=20.0$ i.e. $X$ fluctuates from 0 to 20 and $R_{\max }=50.0$, as $R \rightarrow \infty$. Along the $X$ and $R$ coordinates, subscripts $i$ and $j$ indicate grid points respectively. The geometrical configuration of finite difference grid spacing has been showed in the Fig. 2. Let $U^{\prime}, V^{\prime}, \theta^{\prime}$ and $\phi^{\prime}$ represents the value of $U, V, \theta$ and $\phi$ at termination of time period respectively. Then by explicit finite difference 


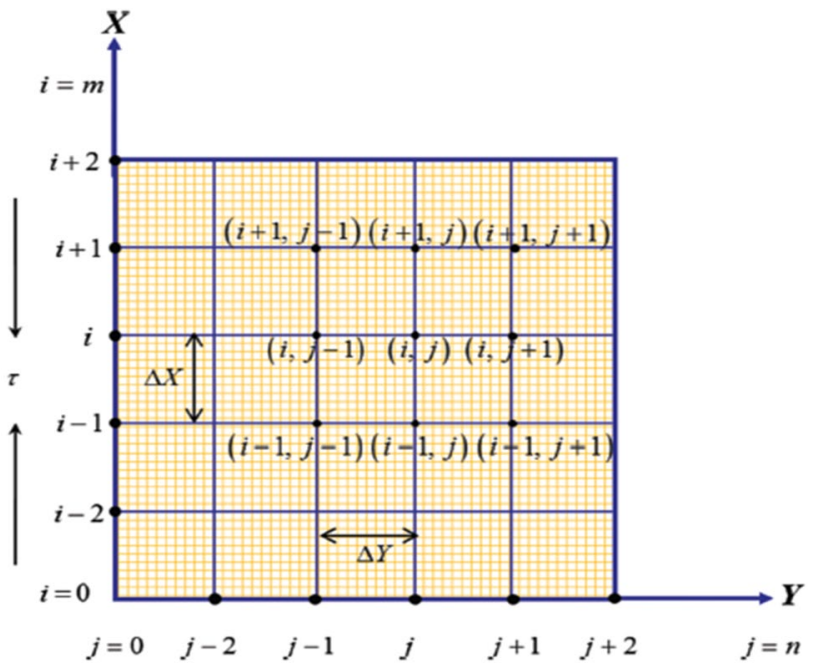

Fig. 2 Finite difference grid space (Rabbi et al. [20])

estimation, it is obtained proper form of continuity, momentum, energy and concentration equations as

$\frac{U(i, j)-U(i-1, j)}{\Delta X}+\frac{V(i, j)-V(i-1, j)}{\Delta R}+\frac{V(i, j)}{1+(j-1) \Delta R}=0$

$\frac{U^{\prime}(i, j)-U(i, j)}{\Delta t}+U(i, j) \frac{U(i, j)-U(i-1, j)}{\Delta X}$

$+V(i, j) \frac{U(i, j+1)-U(i, j)}{\Delta R}=(1-n)$

$\left[\frac{U(i, j+1)-2 U(i, j)+U(i, j-1)}{(\Delta R)^{2}}+\frac{1}{1+(j-1) \Delta R} \frac{U(i, j+1)-U(i, j)}{\Delta R}\right]$

$+W e\left[\frac{U(i, j+1)-U(i, j)}{\Delta R} \times \frac{U(i, j+1)-2 U(i, j)+U(i, j-1)}{(\Delta R)^{2}}+\frac{1}{2(1+(j-1) \Delta R)}\left(\frac{U(i, j+1)-U(i, j)}{\Delta R}\right)^{2}\right]$

$+\operatorname{Gr} \theta(i, j)+G m \phi(i, j)-M U(i, j)$

$$
\begin{aligned}
& \frac{\phi^{\prime}(i, j)-\phi(i, j)}{\Delta t}+U(i, j) \frac{\phi(i, j)-\phi(i-1, j)}{\Delta X} \\
& +V(i, j) \frac{\phi(i, j+1)-\phi(i, j)}{\Delta R} \\
& =\frac{1}{L e}\left[\frac{\phi(i, j+1)-2 \phi(i, j)+\phi(i, j-1)}{(\Delta R)^{2}}+\frac{1}{1+(j-1) \Delta R} \frac{\phi(i, j+1)-\phi(i, j)}{\Delta R}\right] \\
& +\left(\frac{N t}{N b}\right)\left(\frac{\theta(i, j+1)-2 \theta(i, j)+\theta(i, j-1)}{(\Delta R)^{2}}+\frac{\theta(i, j+1)-\theta(i, j)}{\Delta R}\right)-K r \phi(i, j)
\end{aligned}
$$

The primary boundary condition with finite difference scheme as

when, $t \leq 0$ then, $U_{j}^{0}=0, T_{j}^{0}=0, C_{j}^{0}=0$, everywhere when, $t>0$ then $U_{j}^{0}=1, T_{j}^{0}=1, C_{j}^{0}=1$, for all $R=1$

$$
U_{j}^{n}=0, T_{j}^{n}=0, C_{j}^{0}=0 \quad \text { as } R \rightarrow \infty
$$

Here it has been considered $M=100$ and $N=200$ in $X$ and $R$ directions respectively. It is assumed that $\Delta X$ and $\Delta R$ are constant sizes along $X$ and $R$ directions respectively and taken as follows $\Delta X=0.202(0 \leq X \leq 20)$ and $\Delta R=0.251(0 \leq R \leq 50)$ with the smaller time step $\Delta t=0.001$.

\section{Stability and convergence analysis}

Due to the implementation of explicit finite difference technique, a stability and convergence study is extremely needed to fulfill our investigation.

$$
\begin{aligned}
& \frac{\theta^{\prime}(i, j)-\theta(i, j)}{\Delta t}+U(i, j) \frac{\theta(i, j)-\theta(i-1, j)}{\Delta X} \\
& +V(i, j) i \frac{\theta(i, j+1)-\theta(i, j)}{\Delta R}=\frac{1}{\operatorname{Pr}\left(1+\frac{4}{3 R a}\right)} \\
& \quad\left[\frac{\theta(i, j+1)-2 \theta(i, j)+\theta(i, j-1)}{(\Delta R)^{2}}+\frac{1}{1+(j-1) \Delta R} \frac{\theta(i, j+1)-\theta(i, j)}{\Delta R}\right] \\
& +N b \frac{\theta(i, j+1)-\theta(i, j)}{\Delta R} * \frac{\phi(i, j+1)-\phi(i, j)}{\Delta R} \\
& +N t\left(\frac{\theta(i, j+1)-\theta(i, j)}{\Delta R}\right)^{2}+Q \theta(i, j)+\operatorname{EcM}\left(U_{i, j}\right)^{2}
\end{aligned}
$$

The continuity equation i.e. Eq. (19) is neglected because $\Delta t$ does not appear in it. The common relations of the Fourier extension for $U, \theta$ and $\phi$ at time $t=0$ are all $e^{i \alpha X} e^{i \beta Y}$ separately from a constant where $i=\sqrt{-1}$. At a time $t=\tau$, these terms become; 


$$
\begin{gathered}
U: \psi(\tau) e^{i \alpha X} e^{i \beta R} \\
\theta: \theta(\tau) e^{i \alpha X} e^{i \beta R} \\
\varphi: \phi(\tau) e^{i \alpha X} e^{i \beta R}
\end{gathered}
$$

When the time phase changes these expressions become

$U: \psi^{\prime}(\tau) e^{i \alpha X} e^{i \beta R}$

$\theta: \theta^{\prime}(\tau) e^{i \alpha X} e^{i \beta R}$

$\varphi: \phi^{\prime}(\tau) e^{i \alpha X} e^{i \beta R}$

Presently substituting the Eqs. (24) and (25) into the momentum, energy and concentration equation, with respect to the coefficient $U$ and $V$ as constant, the momentum, energy and concentration equations are gained as;

$$
\begin{aligned}
\theta^{\prime} & =\theta\left[1-U \Delta \tau \frac{\left(1-e^{-i \alpha \Delta X}\right)}{\Delta X}-V \Delta \tau \frac{\left(e^{i \beta \Delta R}-1\right)}{\Delta R}\right. \\
& +\frac{1}{P_{r}}\left(1+\frac{4}{3 R_{a}}\right)\left\{\frac{2(\cos \beta \Delta R-1)}{(\Delta R)^{2}}+\frac{1}{R} \frac{\left(e^{i \beta \Delta R}-1\right)}{\Delta R}\right\} \\
& +N b \Delta \tau \frac{\theta\left(e^{i \beta \Delta R}-1\right)}{\Delta R} i \frac{\phi\left(e^{i \beta \Delta R}-1\right)}{\Delta R} \\
& +N t \Delta \tau \phi i\left\{\frac{\theta\left(e^{i \beta \Delta R}-1\right)}{\Delta R}\right\}^{2}+Q \theta \Delta \tau
\end{aligned}
$$

$\theta^{\prime}=A_{4} \theta$

where,

$$
\begin{aligned}
& \frac{\left(\psi^{\prime}-\psi\right) e^{i \alpha x} e^{i \beta R}}{\Delta \tau}+U i \frac{\left(1-e^{-i \alpha \Delta x}\right) e^{i \alpha x} e^{i \beta R}}{\Delta X}+V \frac{\psi\left(e^{i \beta R}-1\right) e^{i \alpha x} e^{i \beta R}}{\Delta R}= \\
& +(1-n)\left\{\frac{2 \psi(\cos \beta \Delta R-1) e^{i \alpha x} e^{i \beta R}}{(\Delta R)^{2}}+\frac{1}{R} \frac{\psi\left(e^{i \beta R}-1\right) e^{i \alpha x} e^{i \beta R}}{\Delta R}\right\} \\
& +W e i\left\{\frac{\psi\left(e^{i \beta R}-1\right) e^{i \alpha x} e^{i \beta R}}{\Delta R}+\frac{1}{2 R} \frac{\psi\left(e^{i \beta R}-1\right) e^{i \alpha x} e^{i \beta R}}{\Delta R}\right\}+G r \theta i+G m \phi i+M \psi i \\
& \psi^{\prime}=\psi\left[1-U \Delta \tau \frac{\left(1-e^{-i \alpha \Delta X}\right) e^{i \alpha x} e^{i \beta R}}{\Delta X}-V \Delta \tau \frac{\left(e^{i \beta R}-1\right) e^{i \alpha x} e^{i \beta R}}{\Delta R}\right. \\
& +(1-n) \Delta \tau\left\{\frac{2(\cos \beta \Delta R-1) e^{i \alpha x} e^{i \beta R}}{(\Delta R)^{2}}+\frac{1}{R} \frac{\left(e^{i \beta R}-1\right) e^{i \alpha x} e^{i \beta R}}{\Delta R}\right\} \\
& W e \Delta \tau\left\{\frac{\left(e^{i \beta R}-1\right) e^{i \alpha x} e^{i \beta R}}{\Delta R} \frac{2(\cos \beta \Delta R-1) e^{i \alpha x} e^{i \beta R}}{(\Delta R)^{2}}+\frac{1}{2 R} \frac{\left(e^{i \beta R}-1\right) e^{i \alpha x} e^{i \beta R}}{\Delta R}\right\} \\
& -M i \Delta \tau]+\operatorname{Gr} \theta \Delta \tau+G m \phi \Delta \tau \\
& \Rightarrow \psi^{\prime}=A_{1} \psi+A_{2} \theta+A_{3} \phi \\
& A_{4}=1-U \Delta \tau \frac{\left(1-e^{-i \alpha \Delta X}\right)}{\Delta X}-V \Delta \tau \frac{\left(e^{i \beta \Delta R}-1\right)}{\Delta R}+\frac{1}{\operatorname{Pr}}\left(1+\frac{4}{3 R_{a}}\right) \\
& \Delta \tau\left\{\frac{(2 \cos \beta \Delta R-1)}{(\Delta R)^{2}}+\frac{1}{R} \frac{\left(e^{i \beta \Delta R}-1\right)}{\Delta R}\right\}+N b \Delta \tau \phi \frac{\left(e^{i \beta \Delta R}-1\right)^{2}}{(\Delta R)^{2}} \\
& +N t \theta \Delta \tau\left(\frac{e^{i \beta \Delta R}-1}{\Delta R}\right)^{2} \\
& \phi^{\prime}=\phi\left[1-U \Delta \tau \frac{\left(1-e^{-i \alpha \Delta X}\right)}{\Delta X}-V \Delta \tau \frac{\left(e^{i \beta \Delta R}-1\right)}{\Delta R}\right. \\
& +\frac{1}{L e}\left\{\frac{2(\cos \beta \Delta R-1)}{(\Delta R)^{2}}+\frac{1}{R} \frac{\left(e^{i \beta \Delta R}-1\right)}{\Delta R}\right\} \\
& \Delta \tau-K r \Delta \tau]+\left(\frac{N t}{N b}\right)\left\{\frac{2(\cos \beta \Delta R-1)}{(\Delta R)^{2}}+\frac{\left(e^{i \beta \Delta R}-1\right)}{\Delta R}\right\} \theta
\end{aligned}
$$

$$
\begin{aligned}
A_{1}= & 1-U \Delta \tau \frac{\left(1-e^{-i \alpha \Delta x}\right) e^{i \alpha x} e^{i \beta R}}{\Delta X}-V \Delta \tau \frac{\left(e^{i \beta R}-1\right) e^{i \alpha x} e^{i \beta R}}{\Delta R} \\
& +(1-n) \Delta \tau\left\{\frac{2(\cos \beta \Delta R-1) e^{i \alpha x} e^{i \beta R}}{(\Delta R)^{2}}+\frac{1}{R} \frac{\left(e^{i \beta R}-1\right) e^{i \alpha x} e^{i \beta R}}{\Delta R}\right\} \\
& W e \Delta \tau\left\{\frac{\left(e^{i \beta R}-1\right) e^{i \alpha x} e^{i \beta R}}{\Delta R} \frac{2(\cos \beta \Delta R-1) e^{i \alpha x} e^{i \beta R}}{(\Delta R)^{2}}+\frac{1}{2 R} \frac{\left(e^{i \beta R}-1\right) e^{i \alpha x} e^{i \beta R}}{\Delta R}\right\}-M \Delta \tau
\end{aligned}
$$

$A_{2}=G r \Delta \tau, \quad A_{3}=G m \Delta \tau$

$$
\phi^{\prime}=A_{5} \theta+A_{6} \phi
$$


where,

$$
\begin{aligned}
& \text { where, } \begin{aligned}
A_{4}= & 1-b_{1}\left(1-e^{-i \alpha \Delta X}\right)-c_{1}\left(e^{i \beta \Delta R}-1\right) \\
& +\frac{1}{\operatorname{Pr}}\left(1+\frac{4}{3 R a}\right)\left\{e_{1}(\cos \beta \Delta R-1)+a_{1}\left(e^{i \beta \Delta R}-1\right)\right\} \\
& +N b \frac{e_{1}}{2}\left(e^{i \beta \Delta R}-1\right)^{2} \phi+N t \frac{e_{1}}{2}\left(e^{i \beta \Delta R}-1\right)^{2} \theta
\end{aligned} \\
& \begin{aligned}
A_{5}=\left(\frac{N t}{N b}\right)\left\{\frac{2(\cos \beta \Delta R-1)}{(\Delta R)^{2}}+\frac{\left(e^{i \beta \Delta R}-1\right)}{\Delta R}\right\} \quad & \left.\left.\frac{1}{R} \frac{\left(e^{i \beta \Delta R}-1\right)}{\Delta R}\right\} \Delta \tau-K r \Delta \tau\right]
\end{aligned}
\end{aligned}
$$

Equations (26), (27), (28) can be presented as matrix form

$$
\left[\begin{array}{c}
\psi^{\prime} \\
\theta^{\prime} \\
\phi^{\prime}
\end{array}\right]=\left[\begin{array}{ccc}
A_{1} & A_{2} & A_{3} \\
0 & A_{4} & 0 \\
0 & A_{5} & A_{6}
\end{array}\right]\left[\begin{array}{l}
\psi \\
\theta \\
\phi
\end{array}\right] \text { i.e. } \eta^{\prime}=T \eta
$$

where,

$$
\eta^{\prime}=\left[\begin{array}{c}
\psi^{\prime} \\
\theta^{\prime} \\
\phi^{\prime}
\end{array}\right], \quad T=\left[\begin{array}{ccc}
A_{1} & A_{2} & A_{3} \\
0 & A_{4} & 0 \\
0 & A_{5} & A_{6}
\end{array}\right], \quad \eta=\left[\begin{array}{l}
\psi \\
\theta \\
\phi
\end{array}\right]
$$

For finite difference study, the dimensionless interval $\Delta \tau$ is minor i.e. inclines to zero. Underneath this complaint, we can write $i A_{2} \rightarrow i 0, i A_{3} \rightarrow i 0, i A_{5} \rightarrow i 0$ and the value of $T$ will be

$T=\left[\begin{array}{ccc}A_{1} & 0 & 0 \\ 0 & A_{4} & 0 \\ 0 & 0 & A_{6}\end{array}\right]$

The Eigen values are $i A_{1}=i \lambda_{1}, i A_{4}=i \lambda_{2}, i A_{6}=i \lambda_{3}$. For stability criteria, the Eigen values do not exceed unity in modulus. Under this criterion the stability situations can be written as,

$\left|A_{1}\right| \leq 1,\left|A_{4}\right| \leq 1,\left|A_{6}\right| \leq 1$

Now for convergence test, let

$a_{1}=\frac{\Delta \tau}{R \Delta R}, b_{1}=\frac{|U| \Delta \tau}{\Delta X}, c_{1}=\frac{|-V \Delta \tau|}{\Delta R}$,

$d_{1}=\Delta \tau, e_{1}=\frac{2 \Delta \tau}{(\Delta R)^{2}}, f_{1}=\frac{\Delta \tau}{\Delta R}$

Then we obtain,

$$
\begin{aligned}
A_{1} & =1-b_{1}\left(1-e^{-i \alpha \Delta X}\right)-c_{1}\left(e^{i \beta \Delta R}-1\right) \\
& +(1-n)\left\{e_{1}(\cos \beta \Delta R-1)+a_{1}\left(e^{i \beta \Delta R}-1\right)\right\} \\
& +W e\left\{f_{1}\left(e^{i \beta \Delta R}-1\right)+\frac{a_{1}}{2}\left(e^{i \beta \Delta R}-1\right)\right\}-M d_{1}
\end{aligned}
$$

$$
\begin{aligned}
A_{5}= & 1-b_{1}\left(1-e^{-i \alpha \Delta X}\right)-c_{1}\left(e^{i \beta \Delta R}-1\right) \\
& +\frac{1}{L e}\left(\left\{e_{1}(\cos \beta \Delta R-1)+a_{1}\left(e^{i \beta \Delta R}-1\right)\right\}-K r d_{1}\right.
\end{aligned}
$$

The possible values are $A_{1}=-1, A_{4}=-1, A_{5}=-1$

$A_{1}=1-2\left[b_{1}+c_{1}+(1-n)\left(e_{1}+a_{1}\right)+W e\left(f_{1}+a_{1}\right)-M \frac{d_{1}}{2}\right.$

$A_{4}=1-2\left[b_{1}+c_{1}+\frac{1}{\operatorname{Pr}}\left(1+\frac{4}{3 R a}\right)\left(e_{1}+a_{1}\right)-N b \phi e_{1}-N t \theta e_{1}\right.$

$A_{5}=1-2\left[b_{1}+c_{1}+\frac{1}{L e}\left(e_{1}+a_{1}\right)-K r \frac{d_{1}}{2}\right]$

Hence the stability conditions are,

$$
\begin{aligned}
& \frac{U \Delta \tau}{\Delta X}+V \frac{\Delta \tau}{\Delta R}+\frac{1}{\operatorname{Pr}}\left(1+\frac{4}{3 R a}\right)\left(\frac{V \Delta \tau}{(\Delta R)^{2}}+\frac{\Delta \tau}{R \Delta R}\right) \\
& -N b \phi \frac{2 \Delta \tau}{(\Delta R)^{2}}-N t \theta \frac{2 \Delta \tau}{(\Delta R)^{2}} \leq 1 \\
& \frac{U \Delta \tau}{\Delta X}+V \frac{\Delta \tau}{\Delta R}+\frac{1}{L e}\left\{\frac{V \Delta \tau}{(\Delta R)^{2}}+\frac{\Delta \tau}{R \Delta R}\right\}+\frac{1}{2} K r \Delta \tau \leq 1
\end{aligned}
$$

With initial boundary conditions $U=V=\theta=\phi=0$ and for $\Delta \tau=0.001, \Delta X=0.202$ and $\Delta R=0.251$ then the model will be congregated at Prandtl number, $\operatorname{Pr} \geq 0.342$ and Lewis number, Le $\geq 0.163$.

Table 1 Skin friction coefficient comparison table $(M=0, W e=0)$

\begin{tabular}{llll}
\hline Le & $\begin{array}{l}\text { Kumar et al. [10] } \\
\text { (RKF-45 method) }\end{array}$ & $\begin{array}{l}\text { Salahuddin et al. [1] } \\
\text { (Shooting method) }\end{array}$ & $\begin{array}{l}\text { Present result } \\
\text { (EFDM) }\end{array}$ \\
\hline 1 & -1.52432 & -1.52432 & -1.52432 \\
8 & -2.47291 & -2.47291 & -2.47295 \\
20 & -3.78263 & -3.78263 & -3.78264 \\
50 & -7.83024 & -7.83024 & -7.83026 \\
100 & -15.58307 & -15.58307 & -15.58308 \\
\hline
\end{tabular}

EFDM explicit finite difference method 
Table 2 Comparison of energy distribution for different values chemical reaction $(K r)$ when $M=0, W e=0, n=0, R=0$

\begin{tabular}{lll}
\hline$K r$ & $\begin{array}{l}\text { Malik et al. [9] } \\
\text { (IFDM) }\end{array}$ & $\begin{array}{l}\text { Presents result } \\
\text { (EFDM) }\end{array}$ \\
\hline 1 & -0.6734 & -0.6742 \\
3 & -1.8426 & -1.8429 \\
5 & - & -1.9373 \\
10 & -2.3809 & -2.3811 \\
100 & - & -7.7560 \\
\hline
\end{tabular}

IFDM implicit finite difference method, EFDM explicit finite difference method

\section{Results and discussion}

Chemically reactive unsteady magnetohydrodynamic two dimensional tangent hyperbolic nanofluid flows with on moving cylinder with Brownian movement and thermophoresis impacts have been investigated. Nanoparticles have higher thermal conductivity and the heat transfer enhancement between these particles can be observed from the point of interaction force. However, by acquiring physical intensity of this issue, the velocity outlines, temperature outlines, concentration outlines, skin friction and Nusselt number outlines are plotted. Moreover, Tables 1, 2 and 3 are exhibited for the validation of the ongoing investigation. The validations of the results are self-evident in the mentioned tables.

Figure 3 represents the illustration of thermal Grashof number $(G r)$ on velocity outlines. It is examined from the figure that velocity field increases with the expanding estimations of thermal Grashof number (Gr). The large value of $G r$ represents the highest velocity and low value of $G r$ represents the lowest velocity. It is perceived, Velocity upsurges at a proportional rate with thermal Grashof number but there is no impact of thermal Grashof number on energy and concentration distributions. Because of increment of thermal buoyancy power which increases the fluid velocity for gravitational strength that helps to

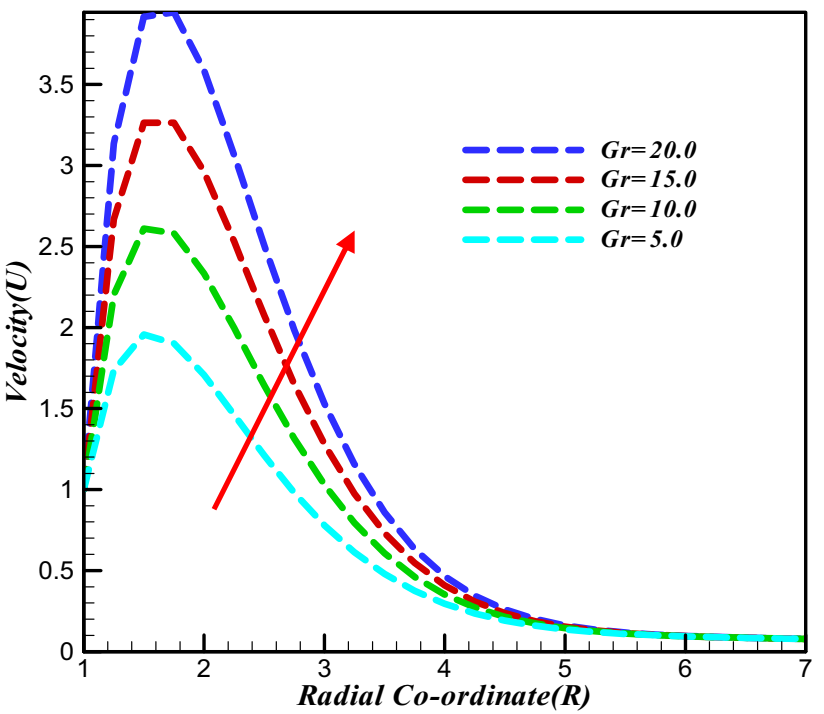

Fig. 3 Variation of $\mathrm{Gr}$ on $U$

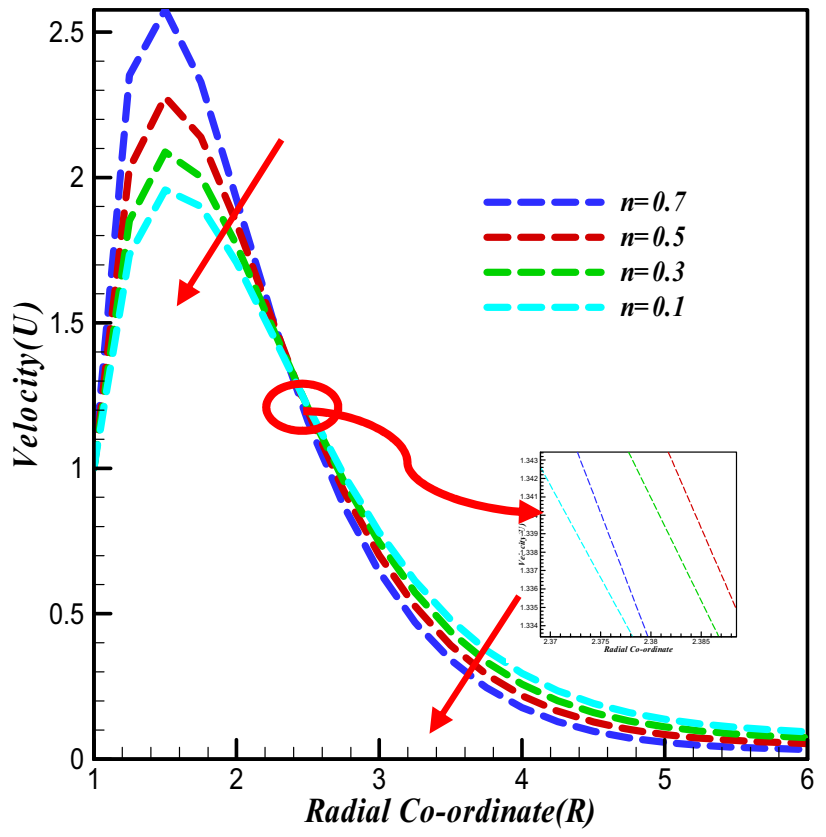

Fig. 4 Variation of $n$ on $U$
Table 3 Graphical comparisons with previous results

\begin{tabular}{|c|c|c|c|c|c|c|c|c|c|}
\hline \multirow[t]{2}{*}{ Factor } & \multicolumn{3}{|c|}{ Hussain et al. [11] } & \multicolumn{3}{|c|}{ Salahuddin et al. [1] } & \multicolumn{3}{|c|}{ Present result } \\
\hline & $\mathrm{U}$ & $\theta$ & $\phi$ & $U$ & $\theta$ & $\phi$ & U & $\theta$ & $\phi$ \\
\hline$M$ & Dec & & & Dec & & & Dec & & \\
\hline Le & & Inc & Dec & Dec & & Dec & Dec & & Dec \\
\hline$Q$ & Inc & & Dec & Inc & $\operatorname{lnc}$ & Dec & Inc & Inc & Dec \\
\hline$N b$ & Inc & Inc & & Inc & Inc & Dec & Inc & Inc & Dec \\
\hline$N t$ & Dec & Inc & Inc & & Inc & Inc & Inc & Inc & Inc \\
\hline We & Dec & Dec & & Inc & & & Dec & & \\
\hline$K r$ & Dec & & & Dec & & Dec & Dec & & Dec \\
\hline
\end{tabular}

Dec decrease, Inc increase 


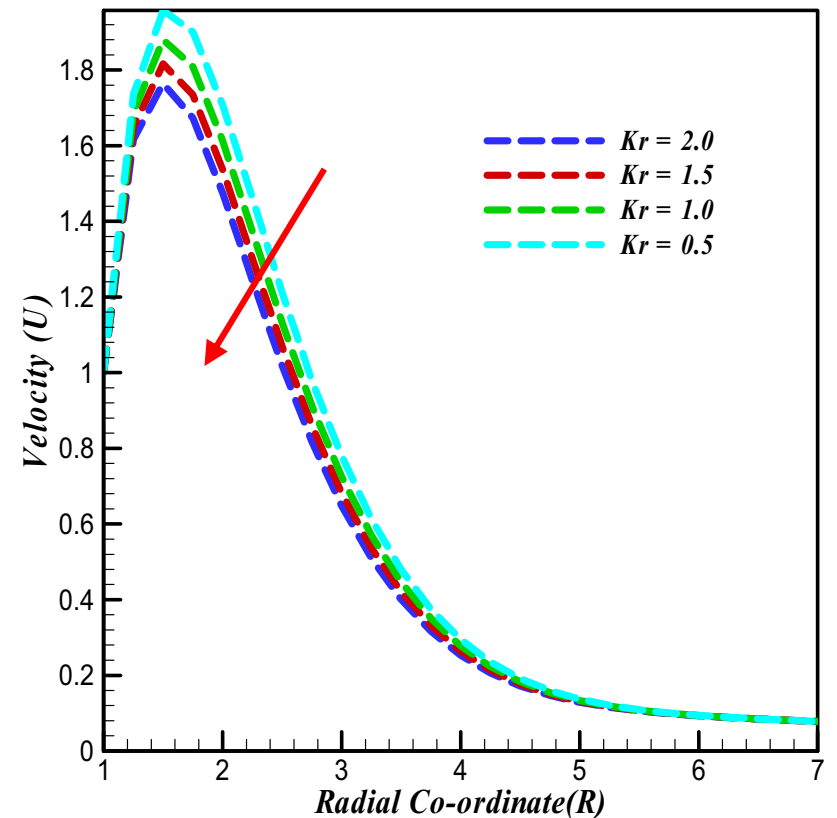

Fig. 5 Variation of $K r$ on $U$

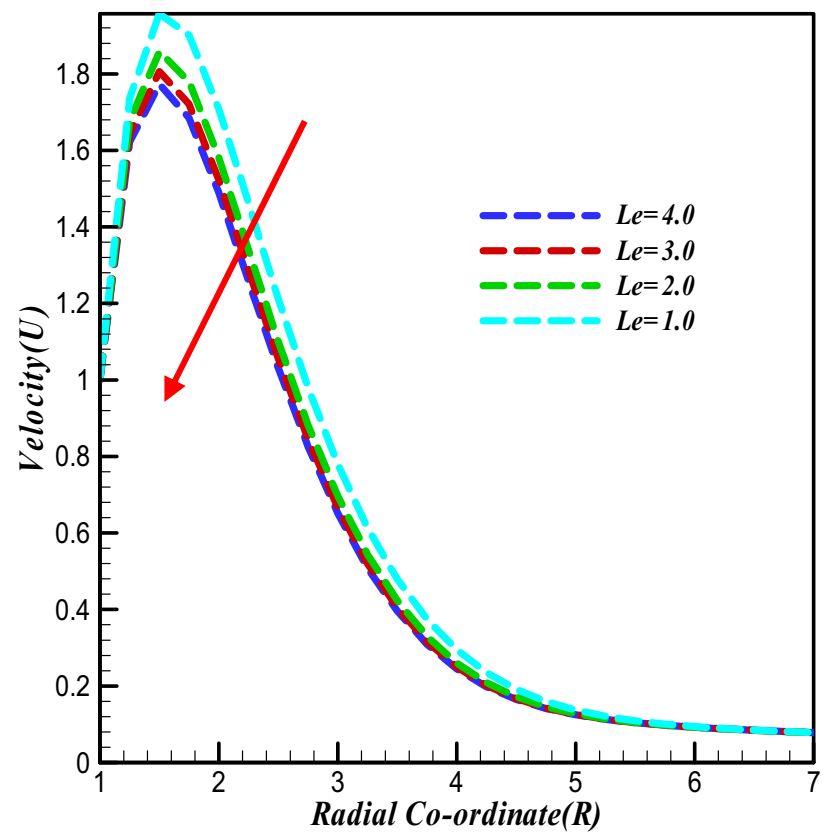

Fig. 6 Variation of Le on $U$

develop the velocity fields. The increasing rate of velocity outline from $G r=5.0$ to $G r=10.0$ is $36.8421 \%, G r=10.0$ to $G r=15.0$ is $26.345 \%$ and $G r=15.0$ to $G r=20.0$ is $18.181 \%$ at $R=2$.

Impacts of different values of power law parameter on velocity dissemination are depicted in the Fig. 4 . In that figure it is perceived that at first velocity upsurges with

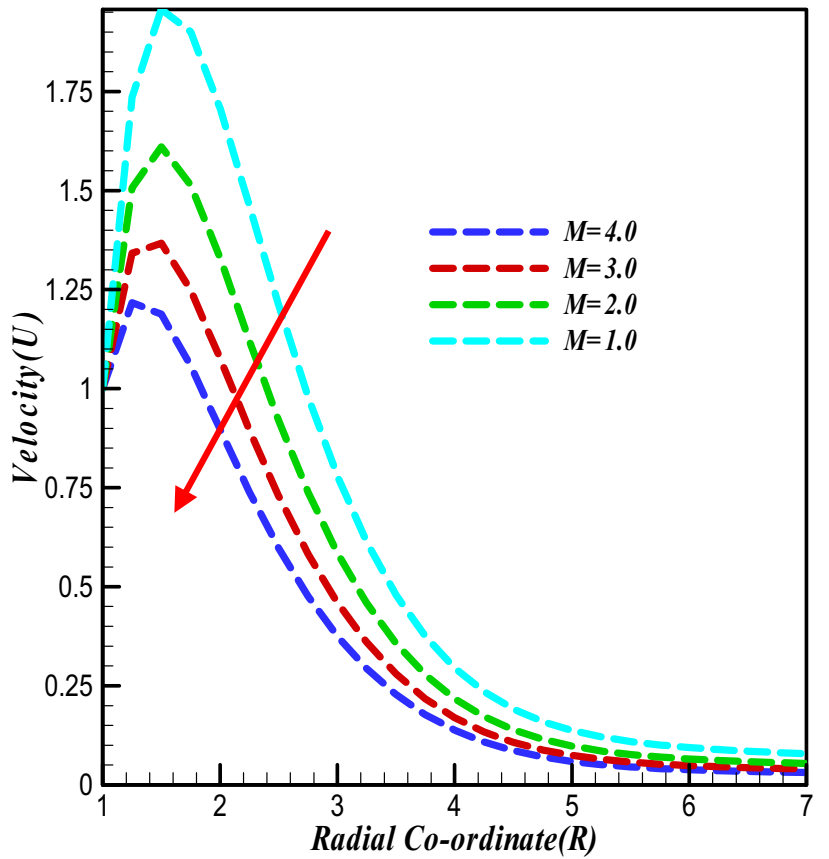

Fig. 7 Variation of $M$ on $U$

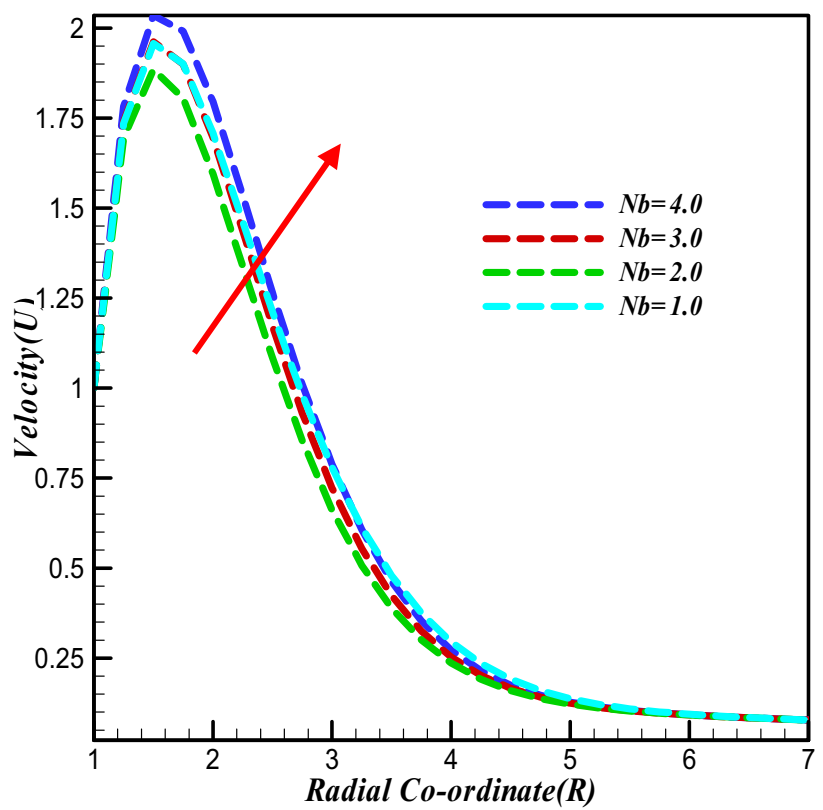

Fig. 8 Variation of $\mathrm{Nb}$ on $\mathrm{U}$

growing value of power parameter index but for a certain value of $R$ the velocity will be equal for all power law indices, probably the value is $R=2.3$ and after a while it is also detected that for increasing of power law parameter decreases the velocity dissemination. The rate of increase of velocity outlines from $n=0.1$ to $n=0.3$ is $10.526 \%$, $n=0.3$ to $n=0.5$ is $9.523 \%$ and $n=0.5$ to $n=0.7$ is $13.043 \%$ 


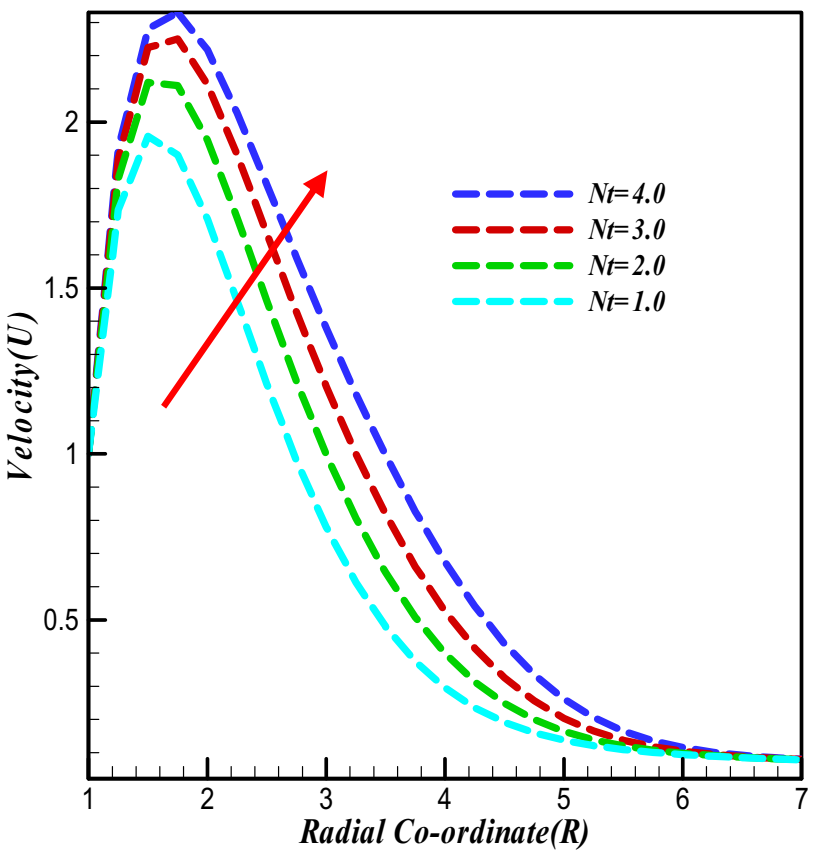

Fig. 9 Variation of $N$ t on $U$

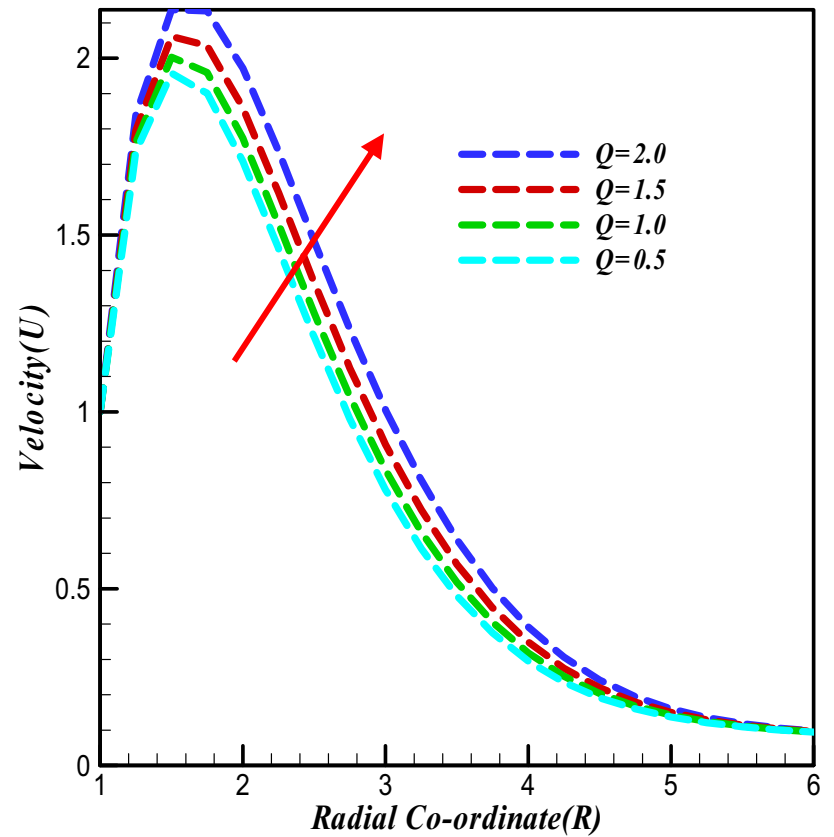

Fig. 10 Variation of $Q$ on $U$

at $R=0.8$ and decreasing rate from $n=0.1$ to $n=0.3$ is $33.333 \%, n=0.3$ to $n=0.5$ is $50.000 \%$ and $n=0.5$ to $n=0.7$ is $48.455 \%$ at $R=4$.

The variation of chemical reaction factor on velocity profile is illustrated in the Fig. 5. Larger values of chemical reaction factor help to reduce the velocity field. Chemical reaction is directly proportional to kinematic viscosity. So

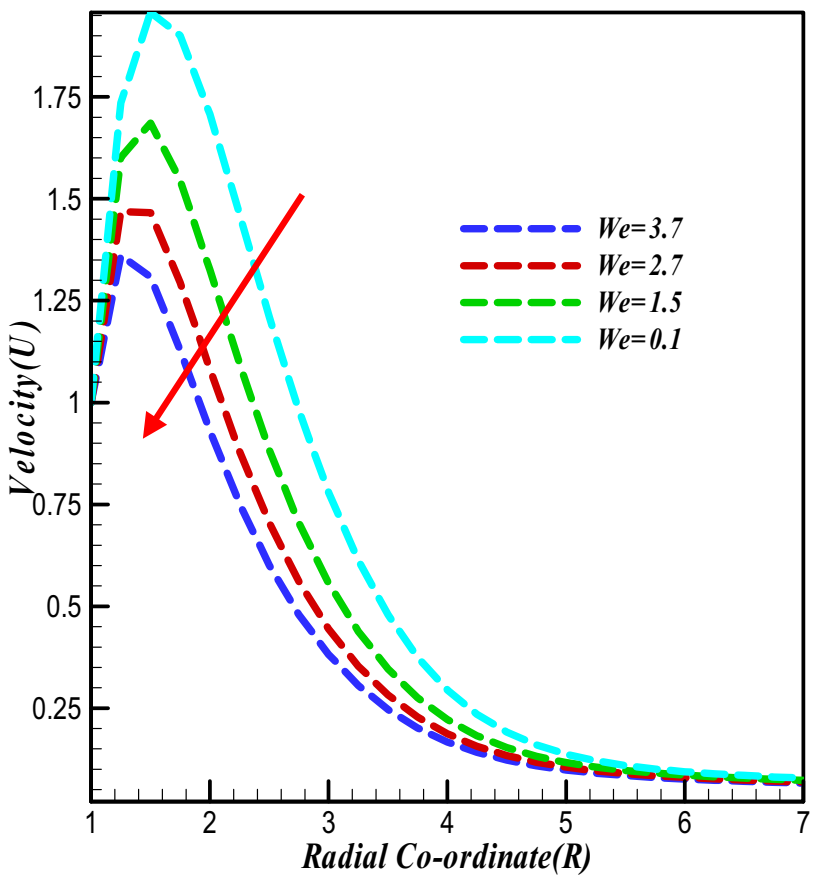

Fig. 11 Variation of We on $U$

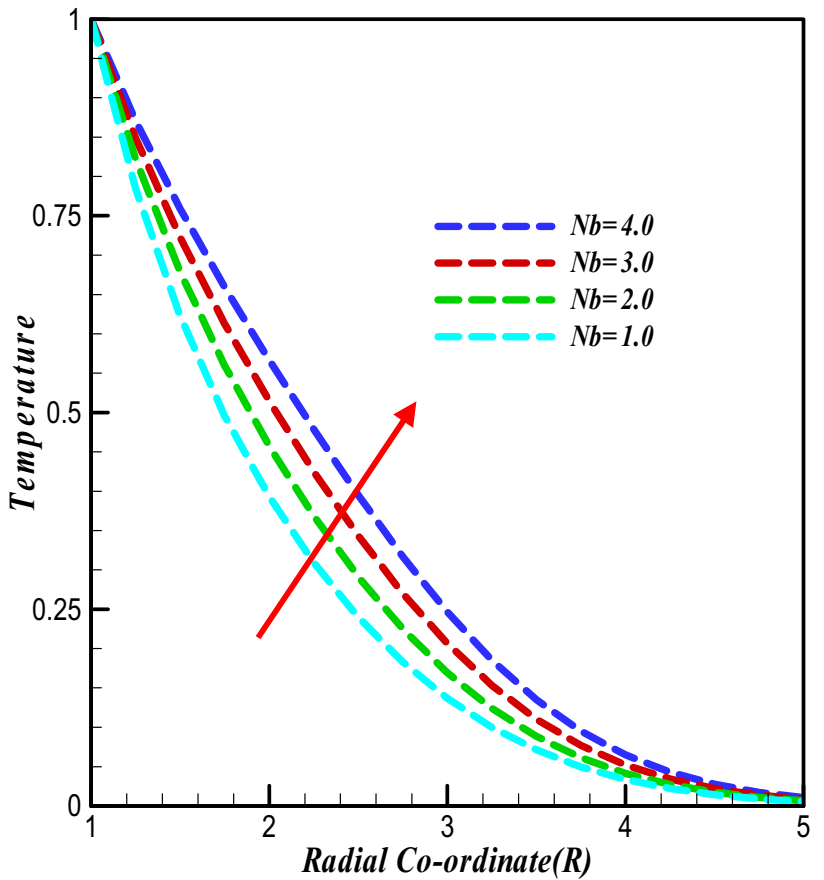

Fig. 12 Variation of $\mathrm{Nb}$ on $\theta$

for the growing of chemical reaction factor, the kinematic viscosity upsurges and the velocity get decrease. The curve to curve decreasing rate of velocity from $K r=0.5$ to $K r=1.0$ is $5.526 \%, K r=1.0$ to $K r=1.5$ is $3.111 \%$ and $K r=1.5$ to $K r=2.0$ is $1.043 \%$ at $R=2.0$. 


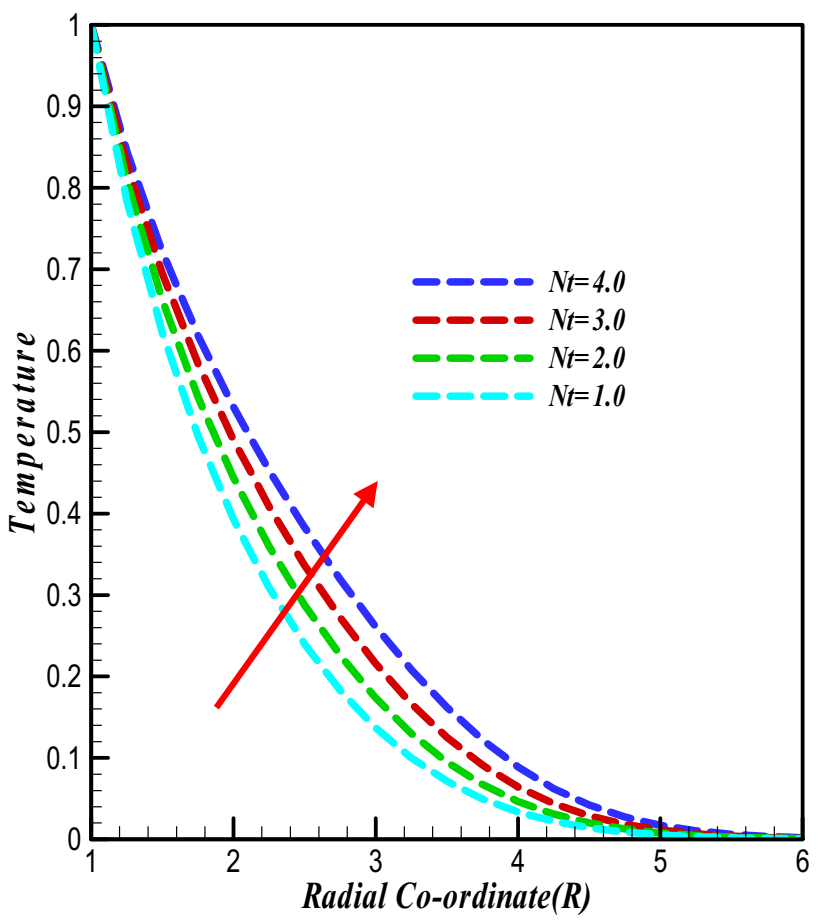

Fig. 13 Variation of $N \operatorname{ton} \theta$

Figure 6 indicates the stimulus of Lewis parameter (Le) on velocity distribution. Lewis parameter $(L e)$ is directly proportional to kinematic viscosity, $v$. When Lewis number upsurges then kinematic viscosity also upsurges. For the reason of increasing of kinematic viscosity the velocity will decrease. The decreasing rate of velocity from $L e=1.0$ to $L e=2.0$ is $5.321 \%, L e=2.0$ to $L e=3.0$ is $2.234 \%$ and $L e=3.0$ to $L e=4.0$ is $1.356 \%$ at $R=2.0$.

The impacts on velocity outline have been exhibited for dissimilar estimation of magnetic factor $(M)$ in Fig. 7. Here the outcomes are perceived that the velocity outline eases with increment of magnetic factor. The reason is that existence of magnetic field creates an obstruction force on the fluid flow. These kinds of power are called Lorentz force which impedes the movement of fluid. The decreasing rate of velocity form curve to curve from $M=1.0$ to $M=2.0$ is $25.321 \%, M=2.0$ to $M=3.0$ is $22.234 \%$ and $M=3.0$ to $M=4.0$ is $14.356 \%$ at $R=0.6$.

The velocity profile for various values of Brownian motion factor $(\mathrm{Nb})$ is depicted in the Fig. 8. The larger particle has weak Brownian motion and for that case the values of $(\mathrm{Nb})$ is small and vice versa. So the increasing value of Brownian factor $(\mathrm{Nb})$ represents the nanoparticle with higher motion. It is detected from figure that velocity distribution upsurges for large value of Brownian motion factor and velocity reduces for its small value. So, the contradiction is that velocity upsurges at a proportional rate with Brownian motion factor. The increasing rate from one

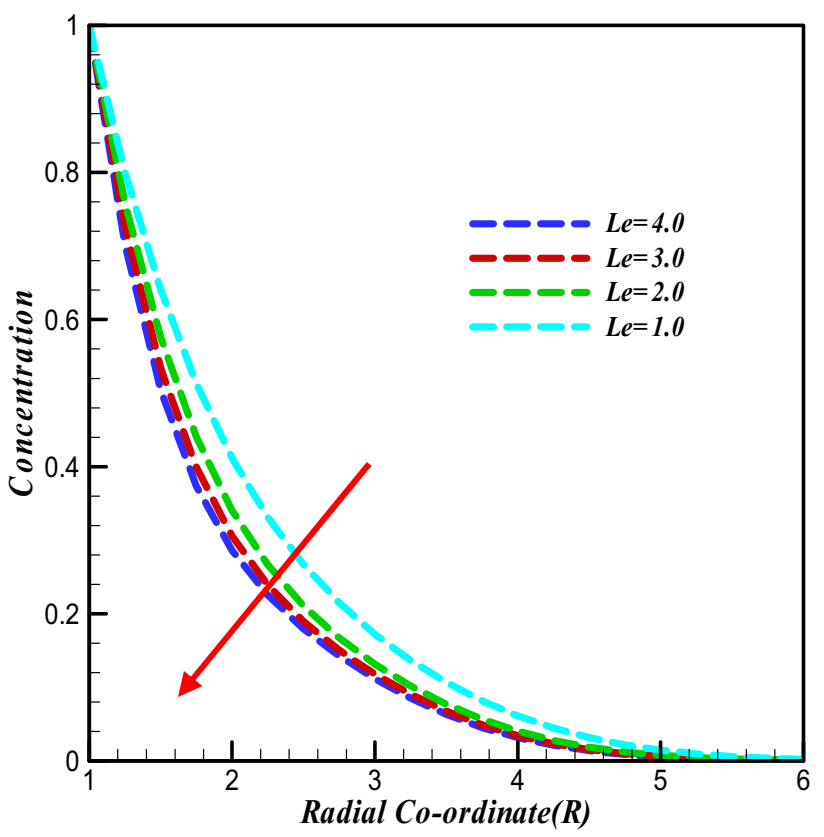

Fig. 14 Variation of Le on $\phi$

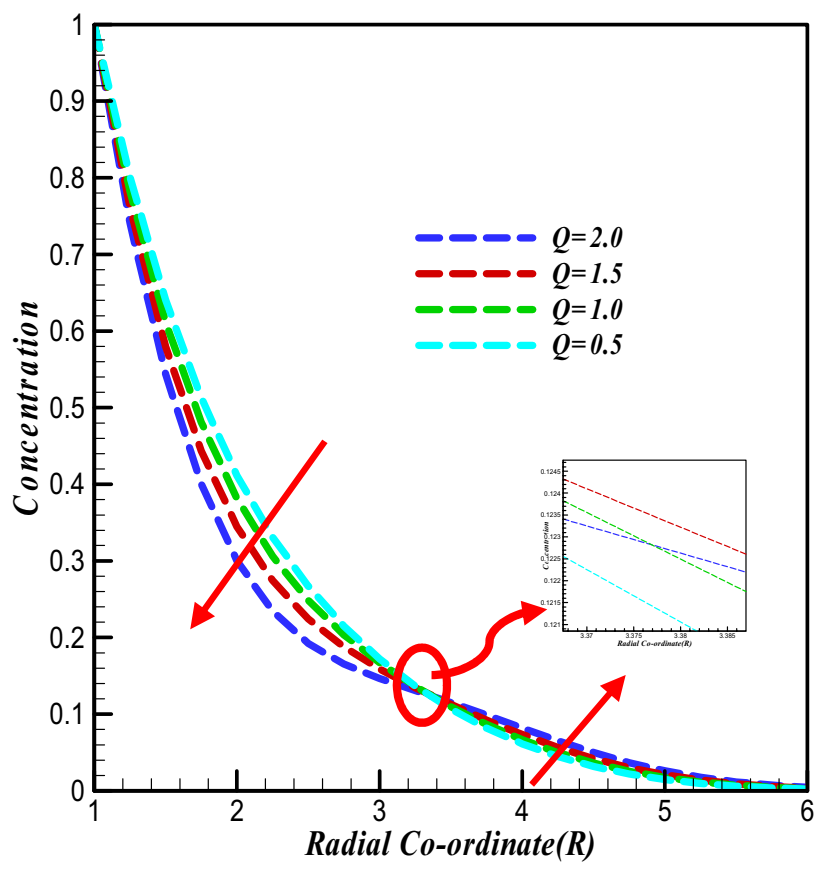

Fig. 15 Variation of $Q$ on $\phi$

curve to other curve is shown with percentage. The rate from $N b=1.0$ to $N b=2.0$ is $3.152 \%, N b=2.0$ to $N b=3.0$ is $0.134 \%$ and $N b=3.0$ to $N b=4.0$ is $4.356 \%$ at $R=2.0$. Figure 9 is depicted for velocity distribution for dissimilar values of thermophoresis number. Thermophoresis factor $(N t)$ usually warms up the boundary layer. So the increasing 

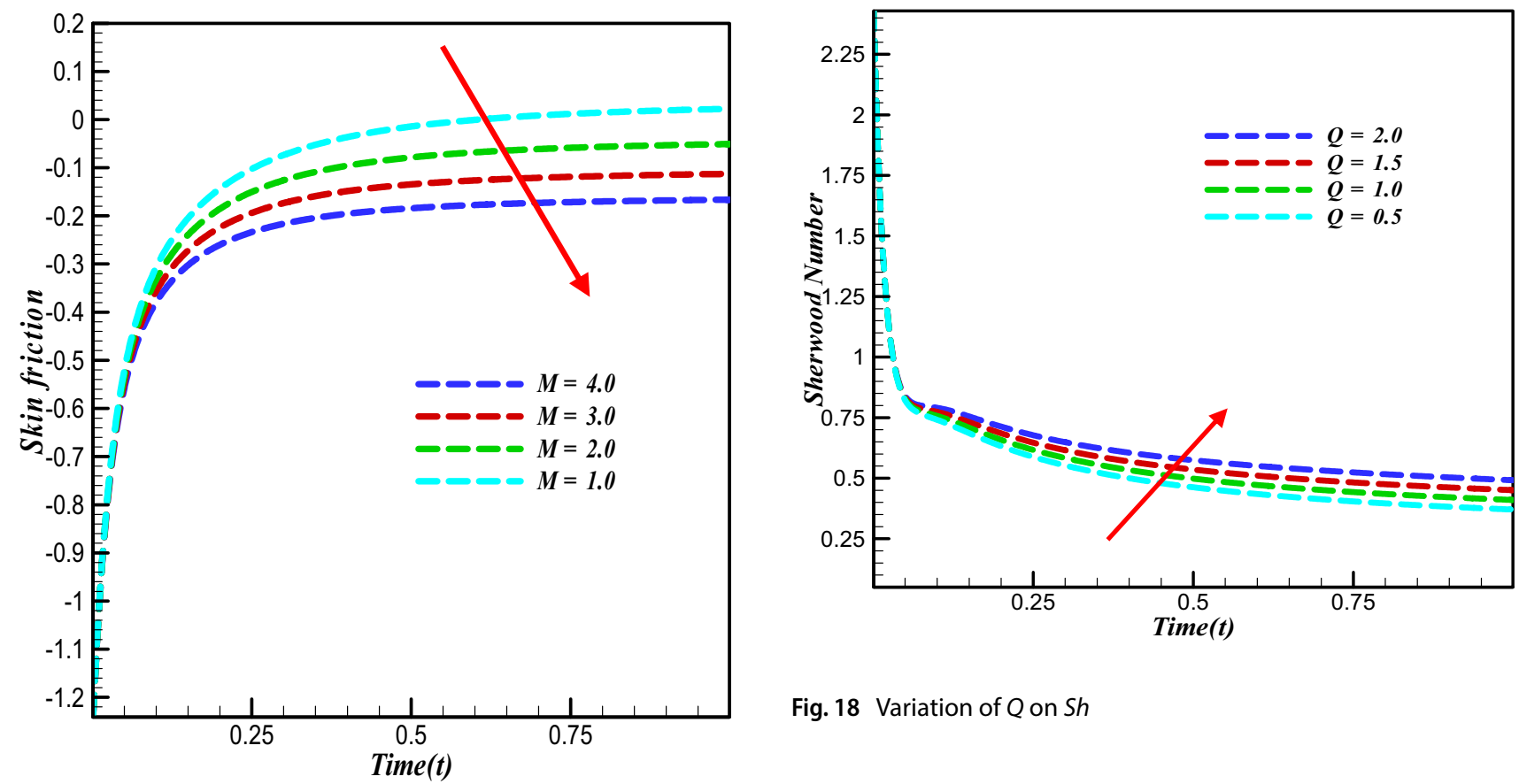

Fig. 16 Variation of $M$ on $C_{f}$

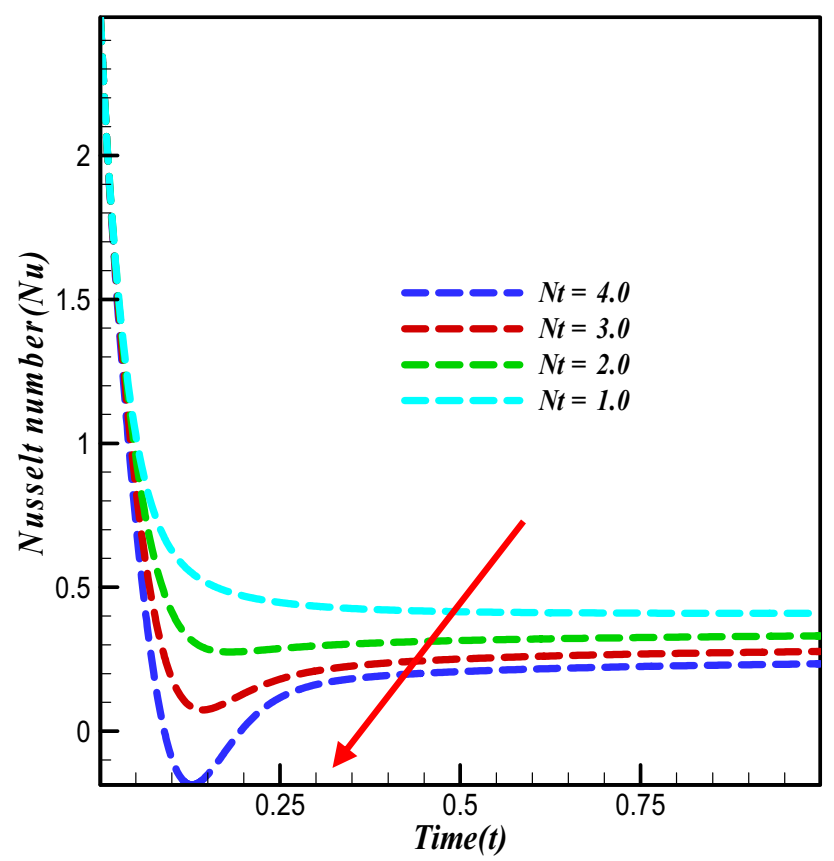

Fig. 18 Variation of $Q$ on $S h$

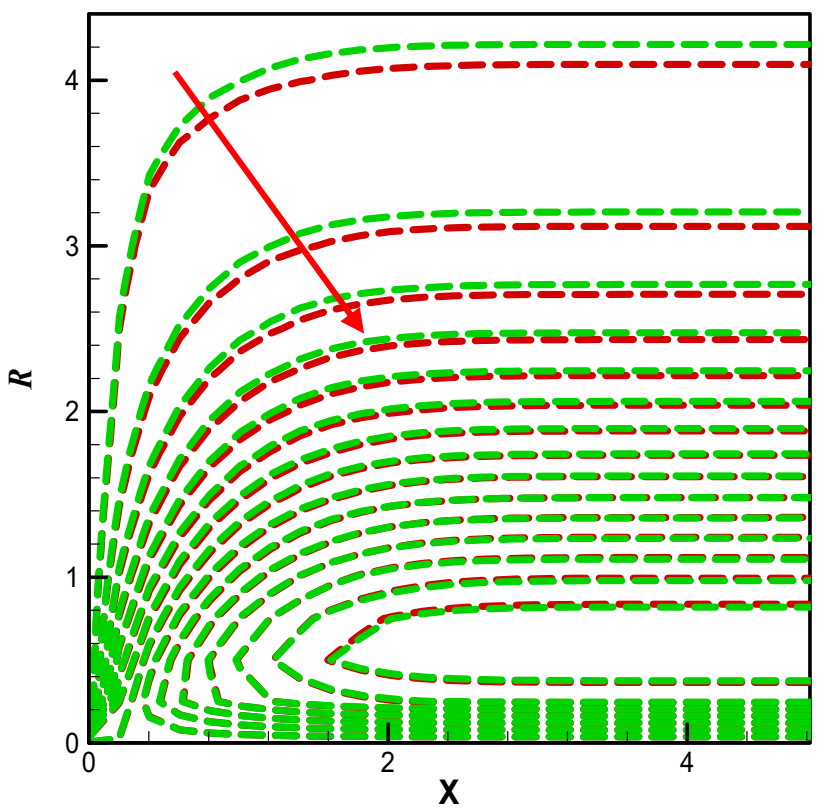

Fig. 19 Streamlines for tangent fluid when $\operatorname{Pr}=1.8$ (red dashed line) and $\operatorname{Pr}=1.2$ (green line)

The impacts of dissimilar values of temperature gen-

Fig. 17 Variation of $\mathrm{Nt}$ on $\mathrm{Nu}$

value of thermophoresis factor, the velocity distribution will improve. Rate of increase from $N t=1.0$ to $N t=2.0$ is $7.152 \%, N t=2.0$ to $N t=3.0$ is $11.34 \%$ and $N t=3.0$ to $N t=4.0$ is $14.356 \%$ at $R=2.0$. eration $(Q)$ on velocity outline are presented in Fig. 10 . It is detected from Fig. 10 that velocity upsurges for the increasing value of heat generation/absorption $(Q)$ factor because increasing value of heat generation/absorption factor upsurges kinematic vitality of the fluid particles. Figure 11 portrays the impacts of Weissenberg number (We) on velocity outline. It is seen from figure, lessening in velocity profile is detected for every growth in the 


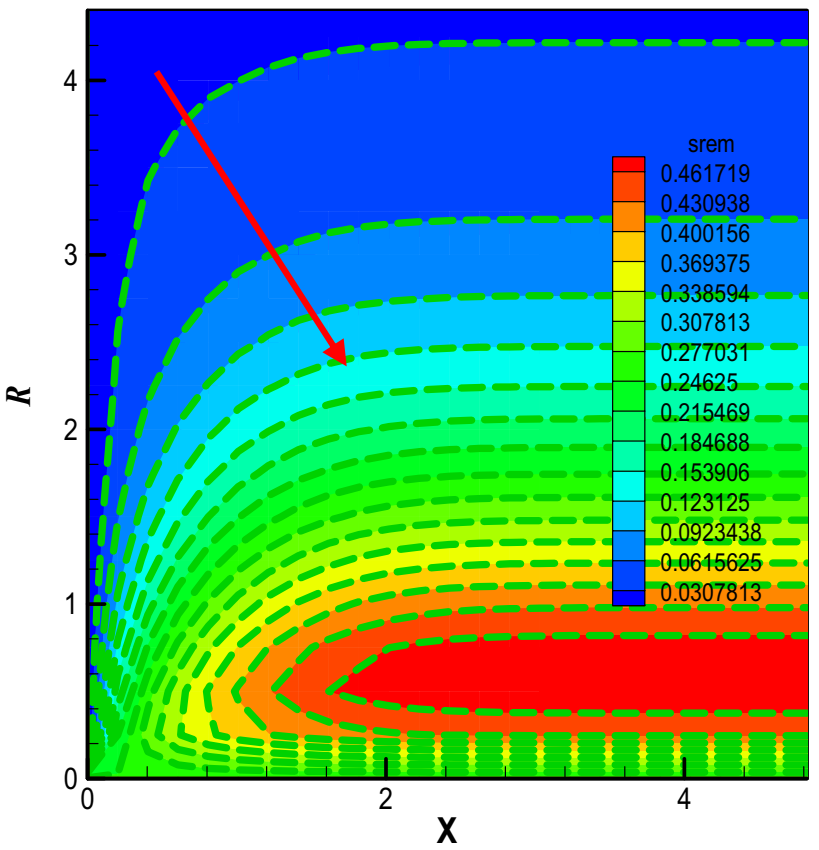

Fig. 20 Streamlines flood view for tangent fluid when $\operatorname{Pr}=1.8$ and $\operatorname{Pr}=1.2$

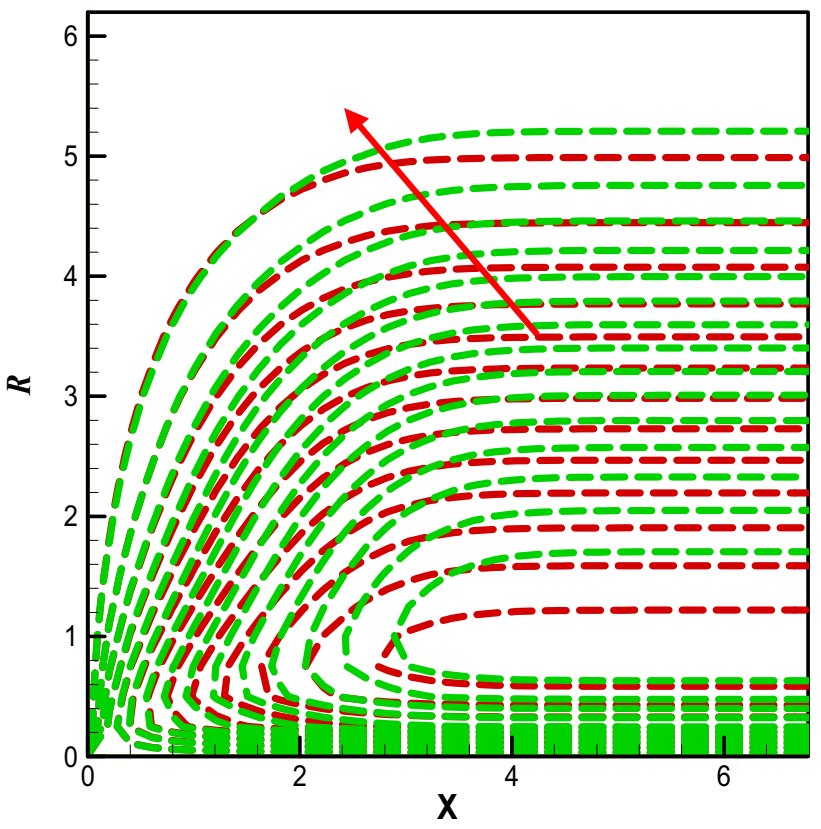

Fig. 21 Streamlines for tangent fluid when $R a=2.5$ (green line) and $R a=2.0$ (red line)

value of Weissenberg number (We), since after growing Weissenberg number (We) the reduction time upsurges which impedes the drift of fluid and henceforth velocity condenses.

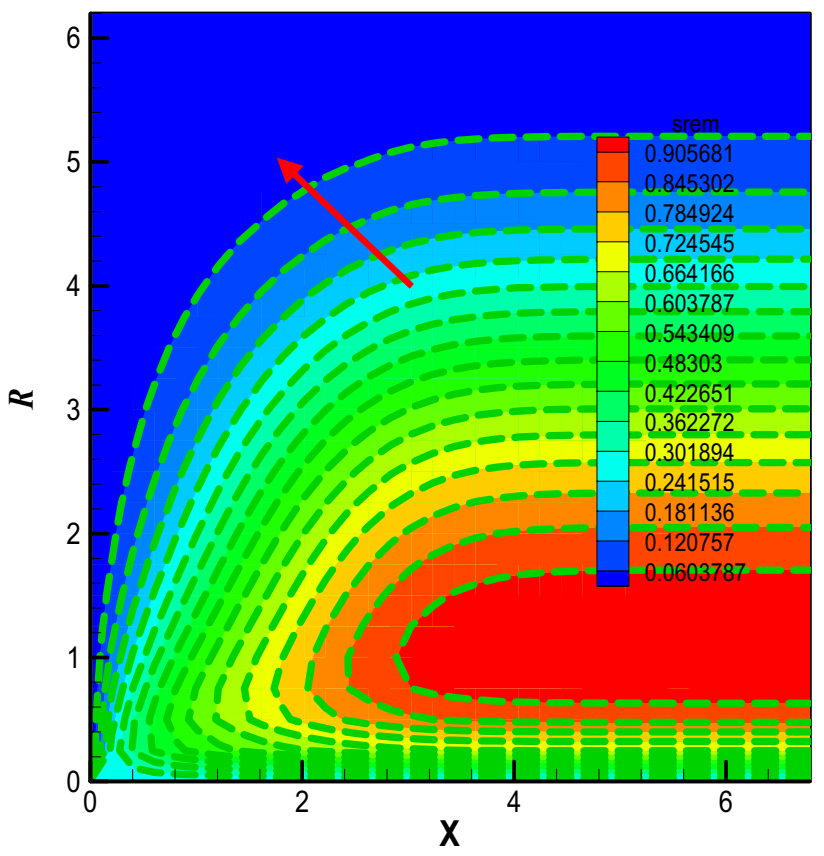

Fig. 22 Streamlines flood view for tangent fluid when $R a=2.5$ and $R a=2.0$

The impacts of Brownian factor $(\mathrm{Nb})$ on temperature outline are introduced in the Fig. 12. It is detected from graph that, since Brownian motion factor $(\mathrm{Nb})$ upsurges the irregular movement of liquid particles the temperature of fluid upsurges. The rate of increment from $\mathrm{Nb}=1.0$ to $N b=2.0$ is $16.152 \%, N b=2.0$ to $N b=3.0$ is $14.134 \%$ and $N b=3.0$ to $N b=4.0$ is $11.111 \%$ at $R=2.1$.

The influence of thermophoresis factor $(\mathrm{Nt})$ on temperature outline is inspected in the Fig. 13. As the values of thermophoresis factor $(\mathrm{N} t)$ upsurges, the liquefied particles transfer from warmer section to cooler section and henceforth velocity of the fluid upsurges. Likewise, the boundary layer upsurges by larger thermophoresis factor Nt.

Figure 14 demonstrates the conduct of Lewis quantity (Le) on concentration outline. It is realized that for expanding the Lewis quantity (Le) the concentration outline decreases. Since for expanding the Lewis number (Le) the mass diffusivity diminishes, so concentration outline diminishes.

The influence of heat generation/absorption factor ( $Q$ ) on concentration outline is depicted by the Fig. 15. It is detected that the increasing value of heat generation/ absorption factor reduces the concentric filed. Due to the divergence of heat flux the fluid velocity increases which declines the fluid concentration and vice versa. The curve to curve decreasing rate is from $Q=0.5$ to $Q=1.0$ is $3.4 \%$, $Q=1.0$ to $Q=1.5$ is $2.454 \%, Q=1.5$ to $Q=2.0$ is $1.345 \%$ at $R=2$. Skin friction for different values of magnetic factor 
$(M)$ has been depicted by the Fig. 16 . It is seen that the skin friction outlines decline for the growing values magnetic factor $(M)$ respectively. Because at the wall, Lorentz force helps to impedes the fluid velocity. The properties of thermophoresis factor on Nusselt number outlines have been shown by the Fig. 17. It is realized from the figure that the Nusselt number declines for growing value of thermophoresis factor. Sherwood number outlines for dissimilar values of heat generation/absorption factor $(Q)$ have been depicted in the Fig. 18. It is seen from figure that the Sherwood number upsurges for the increasing values of generation/absorption factor $(Q)$. The curve increasing rate from $Q=0.5$ to $Q=1.0$ is $12.16 \%, Q=1.0$ to $Q=1.5$ is $16.49 \%$ and $Q=1.5$ to $Q=2.0$ is $22.20 \%$.

The dimensionless equation has been solved by using different transformations. This is why, $X$ and $R$ alliance are dimensionless that indicate mesh theme altered from the arithmetical argument of view. Additionally, streamlines outlines represent improve visualizations of fluid fields. It shows velocity course of fluids correspondingly. The stream lines can be gained by illustration tangent line to the decorations. The boundary layer structure of variation can be presented by an isotherm, where the temperature can be constant Streamlines are illustrated by the Figs. 19, 20, 21 and 22. The Fig. 19 shows the line view of streamlines for $\operatorname{Pr}=1.8$ and $\operatorname{Pr}=1.2$ and the Fig. 20 shows the flood view of streamlines for $\operatorname{Pr}=1.8$ and $\operatorname{Pr}=1.2$. It is detected from the streamlines that the streamlines decrease for large values of Prandtl number because for Prandtl number the thermal conductivity is inversely proportional to the kinematic viscosity.

Figures 21 and 22 depict the streamlines for radiation factor when $R a=2.5$ and $R a=2.0$ and the streamlines are increasing for increasing value of radiation parameter. Because radiation parameter helps to accelerate the divergence of heat flux which eventually increase the fluid velocity as well as the temperature.

\section{Conclusions}

The motivation of this investigation is to do the numerical explanations of time subservient incompressible tangent hyperbolic liquid with nanoparticle concerning a moving cylinder. The impressions of destructive chemical reaction, heat source and thermal radiation were also taken under consideration. The dimensionless forms of the fundamental equations were solved numerically through the implementation of explicit finite difference technique. The default numerical values considered for carrying out the whole investigation are $n=0.1, W e=0.1$,
$G r=5.0, G m=5.0, M=1.0, N b=1.0, N t=1.0, \operatorname{Pr}=1.2$, $R a=0.2, Q=0.5, L e=1.0, K r=0.5$ and $P=1.0$. The work is an extension of the work of Salahuddin et al. [1] and Hayat et al. [7]. The physical impacts of chemical reaction parameter, thermal radiation and viscous dissipation are explored newly in this work. The main concern was how the tangent hyperbolic nanofluid behaves when the above discussed physical impacts are considered in the flow. Again, another concern was to exhibit the developed visualisation of the fluid flow, which has also been illustrated newly in this paper through streamlines. However, for future studies the streamlines and isotherm analysis can be extended for the cases of those parameters which actually appear due to the presence of nanoparticles. Also, to know the different behavior of nanoparticle interaction this work can be extended by considering the magnetic field in sinusoidal form rather than using the usual magnetic term. Moreover, it is a theoretical investigation and more accurate investigation can be done with the implementation of RungeKutta based shooting technique. The major findings of this work are:

The velocity fields exhibit the accelerating attitude when the values of Grashof number, thermophoretic, heat source and Brownian parameters get increase whilst the concentric and the velocity fields got reduce due to the development of chemical reaction, Lewis number, Weissenberg number, magnetic and heat source parameters. It has been also noticed that the interaction between the nanoparticles upsurge the temperature field significantly. However, the skin friction and the heat transfer near the wall get reduce for magnetic and thermophoretic parameters whereas the mass transportation near the wall increases for heat source parameter. Additionally, Prandtl number exhibits the reducing behavior in the depiction of streamlines whilst radiation parameter represent opposite behavior.

\section{Compliance with ethical standards}

Conflict of interest The authors declare that they have no known competing for financial interests or personal relationships that could have appeared to influence the work reported in this paper.

\section{Appendix}

For grid independency test, we have carried out the solutions for different $m$ and $n$. From Fig. 23, we notice that after $m=n=120$ the profiles exert a little change. Therefore, the present results are grid independent. 


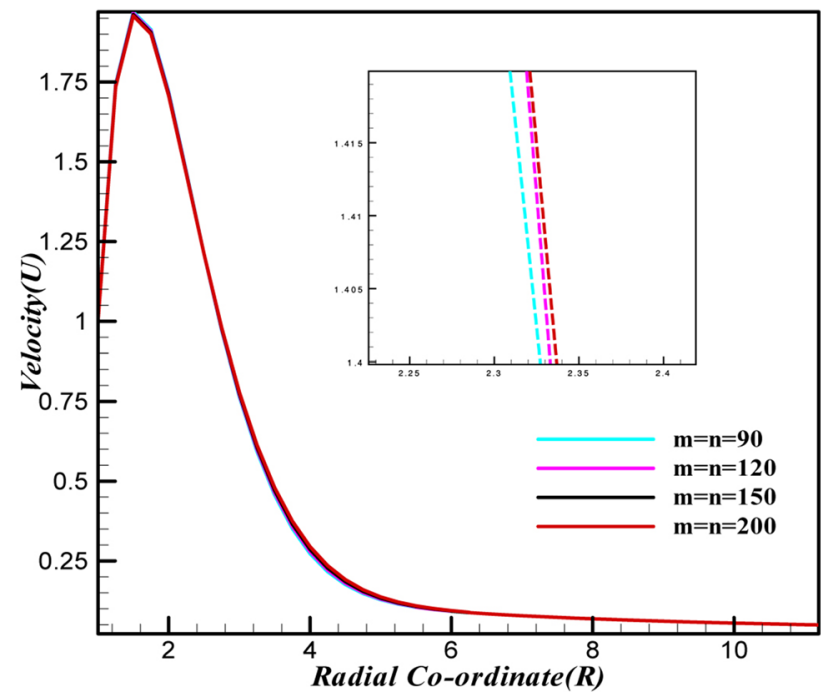

Fig. 23 Grid independency test

\section{References}

1. Salahuddin T, Malik MY, Hussain A, Awis M, Khan I, Khan M (2017) Analysis of tangent hyperbolic nanofluid impinging on a stretching cylinder near the stagnation point. Results Phys 7:426-434

2. Ibrahim W (2017) Magneto hydrodynamics (MHD) flow of a tangent hyperbolic fluid with nanoparticles past a stretching sheet with second order slip and convective boundary condition. Results Phys 7:3723-3731

3. Khan M, Hussain A, Malik MY, Salahuddin T, Khan F (2017) Boundary layer flow of MHD tangent hyperbolic nanofluid over a stretching sheet: a numerical investigation. Results Phys 7:2837-2844

4. Hayat T, Khan MI, Waqas M, Alsaedi A (2017) Stagnation point flow of hyperbolic tangent fluid with Soret-Dufour effects. Results Phys 7:2711-2717

5. Hayat T, Aslam N, Alsaedi A, Rafiq M (2017) Endoscopic effect in MHD peristaltic activity of hyperbolic tangent nanofluid. Results Phys 115:1033-1042

6. Khan M, Malik MY, Salahuddin T (2017) Heat generation and solar radiation effects on Carreau nanofluid over a stretching sheet with variable thickness: using coefficients improved by cash and Crap. Results Phys 7:2512-2519

7. Hayat T, Waqas M, Alsaedi A, Bashir G, Alzaharani F (2017) Magnetohydrodynamic(MHD) stretched flow of tangent hyperbolic nanofluid with variable thickness. J Mol Liq 229:178-184

8. Kumar KG, Gireesha BJ, Krishnamurthy MR, Rudraswamy NG (2017) An unsteady squeezed flow of a tangent hyperbolic fluid over a sensor surface in the presence of variable thermal conductivity. Results Phys 7:3031-3036

9. Malik MY, Salahuddin T, Hussain A, Bilal S (2015) MHD flow of tangent hyperbolic fluid over a stretching cylinder. J Magn Magn Mater 395:271-276

10. Kumar GK, Gorla RSR, Gireesha BJ (2018) Flow and heat transfer of dusty hyperbolic tangent fluid over a stretching sheet in the presence of thermal radiation and magnetic field. Int J Mech Mater Eng 13(2):1-11
11. Hussain A, Malik MY, Salahuddin T, Rubab A, Khan M (2017) Effects of viscous dissipation on MHD tangent hyperbolic fluid over a nonlinear stretching sheet with convective boundary conditions. Results Phys 7(8):3502-3509

12. Khan M, Malik MY, Salahuddin T, Hussain A (2018) Heat and mass transfer of Williamson naofluid flow yield by an inclined Lorentz force over a nonlinear stretching sheet. Results Phys 8:862-868

13. Rahman MM, Al-Lawatia MA, Eltayeb IA, Al-Salti N (2012) Hydro magnetic slip flow of water based nanofluids past a wedge with convective surface in the presence of heat generation (or) absorption. Int J Therm Sci 57:172-182

14. Hamid A, Khan M, Khan U (2018) Thermal radiation effects on Williamson fluid flow due to an expanding/contracting cylinder with nanomaterials. Phys Lett A 382(30):1982-1991

15. Sulochana C, Ashwinkumar GP, Sandeep N (2016) Transpiration effect on stagnation-point flow of a Carreau nanofluid in the presence of thermophoresis and Brownian motion. Alex Eng $J$ 55(4):1151-1157

16. Mamatha SU, Mahesha, Raju CSK (2017) Cattaneo-Christov on heat and mass transfer of unsteady Eyring Powell dusty nanofluid over sheet with heat and mass flux conditions. Inform Med Unlocked 9:76-85

17. Hari RK, Harshad RP (2016) Radiation and chemical reaction effects on MHD Casson fluid flow past an oscillating vertical plate embedded in porous medium. Alex Eng J 55:583-595

18. Reza-E-Rabbi Sk, Arifuzzaman SM, Sarkar T, Khan M, Ahmmed SF (2020) Explicit finite difference analysis of an unsteady MHD flow of a chemically reacting Casson fluid past a stretching sheet with Brownian Motion and thermophoresis effects. J King Saud Univ Sci 32(1):690-701

19. Arifuzzaman SM, Khan MS, Al-Mamun A, Reza-E-Rabbi Sk, Biswas P, Karim I (2019) Hydrodynamic stability and heat and mass transfer flow analysis of MHD radiative fourth-grade fluid through porous plate with chemical reaction. J King Saud Univ Sci 31(4):1388-1398

20. Reza-E-Rabbi S, Ahmmed SF, Sarker T, Arifuzzaman SM, Khan M (2020) Computational modeling of multiphase fluid flow behavior over a stretching sheet in presence of nanoparticles. Eng Sci Technol Int J 23(3):605-617

21. Ma X, Sheikholeslami M, Jafaryar M, Shafee A, Nguyen-Thoi T, Li $Z$ (2019) Solidification inside a clean energy storage unit utilizing phase change material with copper oxide nanoparticles. J Clean Prod 245:118888

22. Hsiao K-L (2017) Combined electrical MHD heat transfer thermal extrusion system using Maxwell fluid with radiative and viscous dissipation effects. Appl Therm Eng 112:1281-1288

23. Sheikholeslami M (2019) New computational approach for exergy and entropy analysis of nanofluid under the impact of Lorentz force through a porous media. Comput Methods Appl Mech Eng 344:319-333

24. Qi C, Liu M, Tang J (2019) Influence of triangle tube structure with twisted tape on the thermohydraulic performance of nanofluids in heat-exchange system based on thermal and exergy efficiency. Energy Convers Manag 192:243-268

25. Zhao N, Qi C, Chen T, Tang J, Cui X (2019) Experimental study on influences of cylindrical grooves on thermal efficiency, exergy efficiency and entropy generation of CPU cooled by nanofluids. Int J Heat Mass Transf 135:16-32

Publisher's Note Springer Nature remains neutral with regard to jurisdictional claims in published maps and institutional affiliations. 